\title{
A missense mutation in Pitx2 leads to early-onset glaucoma via NRF2-YAP1 axis
}

\author{
Yeming Yang ${ }^{1,2,3,7}$, Xiao $\mathrm{Li}^{1,7}$, Jieping Wang ${ }^{4}$, Junkai Tan (iD) ${ }^{5}$, Bernie Fitzmaurice ${ }^{4}$, Patsy M. Nishina ${ }^{4}$, Kuanxiang Sun ${ }^{1}$, Wanli Tian ${ }^{1}$, \\ Wenjing Liu ${ }^{1}$, Xuyang Liu ${ }^{5,6 \bowtie}$, Bo Chang (iD ${ }^{4 凶}$ and Xianjun Zhu (iD ${ }^{1,2,3 凶}$
}

(c) The Author(s) 2021

Glaucoma is a leading cause of blindness, affecting 70 million people worldwide. Owing to the similarity in anatomy and physiology between human and mouse eyes and the ability to genetically manipulate mice, mouse models are an invaluable resource for studying mechanisms underlying disease phenotypes and for developing therapeutic strategies. Here, we report the discovery of a new mouse model of early-onset glaucoma that bears a transversion substitution c. G344T, which results in a missense mutation, p. R115L in PITX2. The mutation causes an elevation in intraocular pressure (IOP) and progressive death of retinal ganglion cells (RGC). These ocular phenotypes recapitulate features of pathologies observed in human glaucoma. Increased oxidative stress was evident in the inner retina. We demonstrate that the mutant PITX2 protein was not capable of binding to Nuclear factor-like 2 (NRF2), which regulates Pitx2 expression and nuclear localization, and to YAP1, which is necessary for co-initiation of transcription of downstream targets. PITX2-mediated transcription of several antioxidant genes were also impaired. Treatment with N-Acetyl-L-cysteine exerted a profound neuroprotective effect on glaucoma-associated neuropathies, presumably through inhibition of oxidative stress. Our study demonstrates that a disruption of PITX2 leads to glaucoma optic pathogenesis and provides a novel early-onset glaucoma model that will enable elucidation of mechanisms underlying the disease as well as to serve as a resource to test new therapeutic strategies.

Cell Death and Disease (2021)12:1017; https://doi.org/10.1038/s41419-021-04331-1

\section{INTRODUCTION}

Glaucoma, affecting 70 million people worldwide, is a group of neurodegenerative diseases characterized by the progressive death of retinal ganglion cells (RGCs) and atrophic excavation of the optic nerve, ultimately resulting in irreversible loss of vision $[1,2]$. Elevated intraocular pressure (IOP) is thought to be a major risk factor for glaucoma, and patients with high-pressure glaucoma generally present defects in outflow of aqueous humor $[3,4]$. IOP is maintained through a balance between aqueous humor secretion, by the ciliary body $(\mathrm{CB})$, and drainage through the trabecular meshwork (TM), a porous tissue located in the iridocorneal angle [5]. An increased resistance to aqueous humor drainage can arise from developmental malformations of ocular structures and ultimately lead to early-onset glaucoma.

Early-onset glaucoma cases generally have strong genetic contributions and many disease-causing mutations that lead to pathogenesis have been identified [6]. For example, mutations in CYP1B1, LTBP2, MYOC, FOXC1, GPATCH3, and TEK have been associated with Primary Congenital Glaucoma (PCG) [7]. Moreover, mutations in PAX6, PITX2, PITX3, FOXC1, FOXE3, EYA1, LMX1B, and $M A F$, have been detected in patients with early-onset secondary glaucoma [8-12]. It should also be noted that glaucoma is a complex, heterogeneous disease likely to be the consequence of the interaction of multiple genes. This underlying complexity may hinder efforts to identify glaucoma-associated genes (or related mutations) and to uncover their pathogenic mechanisms. Identification of monogenic murine glaucoma models, may assist in addressing both of these issues. To this end, the Eye Mutant Resource screening program at The Jackson Laboratory (JAX) identified a glaucoma model caused by a mutation in Pitx2.

In humans, mutations in Paired-Like Homeodomain Transcription Factor 2 (PITX2) has been associated with Axenfeld-Rieger syndrome (ARS) (OMIM: 180500). PITX2, which encodes a member of the bicoid-like class of homeodomain (HD) transcription factors, play key roles in embryonic development and tissue morphogenesis. To date, a range of PITX2 mutations have been identified in ARS patients including missense, nonsense, splicing mutations, and copy number variations $[13,14]$. The majority of ARS-causing mutations are missense mutations within the HD region, which may impair DNA binding and decreasing transactivation activity [15]. ARS in humans is characterized by both systemic and ocular anomalies, such as corneal opacity, iris hypoplasia, corectopia, and

\footnotetext{
${ }^{1}$ Sichuan Provincial Key Laboratory for Human Disease Gene Study, Center for Medical Genetics, Sichuan Provincial People's Hospital, University of Electronic Science and Technology of China, 610072 Chengdu, Sichuan, China. ${ }^{2}$ Henan Eye Institute, Henan Eye Hospital, People's Hospital of Zhengzhou University, Henan Provincial People's Hospital, 450003 Zhengzhou, Henan, China. ${ }^{3}$ Research Unit for Blindness Prevention of Chinese Academy of Medical Sciences (2019RU026), Sichuan Academy of Medical Sciences \& Sichuan Provincial People's Hospital, 610072 Chengdu, Sichuan, China. ${ }^{4}$ The Jackson Laboratory, Bar Harbor, ME 04609 , USA. ${ }^{5}$ Xiamen Eye Center, Xiamen University, 361006 Xiamen, Fujian, China. ${ }^{6}$ Department of Ophthalmology, Shenzhen People's Hospital, the 2nd Clinical Medical College, Jinan University, 518020 Shenzhen, China. ${ }^{7}$ These authors contributed equally: Yeming Yang, Xiao Li. ${ }^{凶}$ email: xliu1213@126.com; bo.chang@jax.org; xjzhu@uestc.edu.cn

Edited by Professor Daniel Aberdam
}

Received: 30 June 2021 Revised: 12 October 2021 Accepted: 18 October 2021

Published online: 29 October 2021 
iridolenticular adhesions [12]. These developmental malformations of the anterior segment in ARS patients lead to severe forms of early-onset glaucoma in $\sim 50 \%$ of affected individuals.

Previous studies have suggested that Pitx2 deficiency in mice lead to an arrest in anterior segment development in structures derived from the periocular mesenchyme, including the cornea, iris and outflow tract, which likely results from abnormal differentiation and migration of neural crest cells during the formation of anterior ocular structures [16-20]. Moreover, mice heterozygous for a Pitx2 null allele recapitulate the anterior segment dysplasia and developmental glaucoma observed in ARS patients [21]. However, the role of PITX2 in maintenance of normal optic function in adults and the precise mechanisms underlying glaucomatous pathologies when PITX2 is disrupted remain largely unknown.

In this study, we identified a novel missense mutation, c. G344T, p. R115L, in Pitx2. In the homozygous state, mice bearing this mutation presents with bulging and distended eyes, a striking feature of glaucoma. Moreover, increased oxidative stress was evident in mutant retinas, which further activated persistent glial activation in the inner retina and RGC apoptosis. Mechanistically, the missense mutation disrupts protein interaction of PITX2 with NRF2 (regulating Pitx2 expression and nuclear localization) and YAP1 (co-initiating transcription of downstream targets), leading to impaired PITX2-mediated transcription of several antioxidant genes, which activates the antioxidant response after ocular injury. Additionally, treatment with N-Acetyl-L-cysteine exhibited profound neuroprotective effect. Overall, this work describes a novel early-onset glaucoma model and sheds light on the pathogenesis of glaucoma in ARS.

\section{MATERIALS AND METHODS \\ Mouse models}

All animal experiments were approved by the Institutional Animal Care and Use Committee of Sichuan Provincial People's Hospital (Chengdu, Sichuan, China) and The Jackson laboratory (JAX, Bar Harbor, Maine, USA) and conducted in accordance with the ARVO Statement for the Use of Animals in Ophthalmic and Vision Research.

The original mutant was identified in a $\mathrm{N}$-ethyl-N-nitrosourea (ENU) chemical mutagenesis screen in the Mouse Resource for Craniofacial Research (http://craniofacial.jax.org/). Briefly, C57BL/6J male mice, G0, were treated with ENU (weekly $80 \mathrm{mg} / \mathrm{kg}$ dosage for 3 weeks), and bred to normal C57BL/6J females after returning to fertility. G1 male offspring were backcrossed to unmutagenized C57BL/6J females and their G2 female offspring were backcrossed to their $\mathrm{G} 1$ sires to generate a population of $\mathrm{G} 3$ mice that were screened for visible phenotypes. A G3 mutant with a shortened face and enlarged eyes was identified. This mutant was backcrossed again to C57BL/6J and the short face phenotype segregated from the ocular phenotype. This mutant was named early-onset glaucoma (eg/1) and was bred to homozygosity and subsequently maintained by sibling intercrosses (C57BL/6J-Pitx2 $2^{\text {eg/7 }} /$ Boc; stock No. 004240). The eg/1 mutant mice in this study were bred and maintained in standardized conditions of the Research Animal Facilities at The Jackson Laboratory (JAX). They were provided with a NIH31 6\% fat chow diet and acidified water, in a pathogen-free vivarium environment with a 14-hour light/10hour dark cycle.

\section{Clinical evaluation and electroretinography}

Eyes of all mice used in the characterization studies and linkage crosses were dilated with $1 \%$ atropine ophthalmic drops (Bausch and Lomb Pharmaceuticals Inc., Tampa, FL, USA) and were evaluated by indirect ophthalmoscopy with a 78-diopter lens. Fundus photographs were taken with a Micron III in vivo bright field retinal imaging microscope equipped with image-guided OCT capabilities (Phoenix Laboratories, Inc, Phoenix, $A Z$, USA). The intraocular pressure (IOP) was measured by an induction-impact tonometer (TonoLab Colonial Medical Supply, Londonderry, $\mathrm{NH}, \mathrm{USA})$. IOP was measured immediately after induction with an intraperitoneal injection of xylazine $(80 \mathrm{mg} / \mathrm{kg})$ and ketamine $(16 \mathrm{mg} / \mathrm{kg})$ in normal saline $(\sim 5 \mathrm{~min})$. The mouse was gently restrained by hand on an adjustable platform, and the eye was oriented to align the probe tip with the optical axis of the eye at about 2-mm distance under dissecting scope. All tested animals with the same age were randomly divided into the experimental groups. Five consecutive IOP readings were averaged.

For electroretinographic evaluation of mutants, following an overnight dark adaptation, mice were anesthetized with an intraperitoneal injection of xylazine $(80 \mathrm{mg} / \mathrm{kg})$ and ketamine $(16 \mathrm{mg} / \mathrm{kg})$ in normal saline. Additional anesthetic was given, if akinesia was inadequate. The equipment and protocol used here have been previously described [22]. Briefly, dark-adapted, rod-mediated ERGs were recorded with the responses to short-wavelength flashes over 4.0-log units to the maximum intensity by a photopic stimulator. Cone-mediated ERGs were recorded with white flashes after $10 \mathrm{~min}$ of complete light adaptation. The signals were sampled at $0.8 \mathrm{~ms}$ intervals and averaged.

\section{Whole-exome sequencing (WES)}

DNA purification, library construction, deep Next Generation sequencing, and data quality control was performed by the Jackson Laboratory's Genome Technologies service, and data analysis and annotation were performed by the Computational Sciences Biostatistics service. Purified genomic DNA from eg/1/eg/1 mice and C57BL/6J controls were used to create libraries for whole-exome sequence (WES) capture. High-quality reads were mapped to the mouse genome (GRCm38, mm10) and the resulting alignment was sorted by coordinates and further converted to binary alignment map (BAM) format by Picard v1.95 SortSam utility (http:// picard.sourceforge.net). Variants with total read depth $>5 \mathrm{X}$ with the SNP quality score $>50$ were included and variants in non-coding regions and synonymous variants were excluded. The filtered candidate genes are shown in Table S1.

\section{Gene mapping, sequencing, and genotyping}

To determine the chromosomal location of the eg/1 mutation, we mated eg/1/eg/1 mice to DBA/2J mice. The resultant F1 mice, which did not exhibit retinal abnormalities, were backcrossed to B6-eg/1/eg/1 mice to produce N2 mice. Tail DNA was isolated as previously reported [23]. A genome-wide scan of pooled DNA from 12 affected and 12 unaffected mice was carried out using 48 microsatellite markers [24]. The eg/1 phenotype cosegregated with markers on Chromosome 3. Subsequently, DNAs of 93 N2 offspring were genotyped using microsatellite markers to develop a fine structure map of the Chromosome 3 region. Microsatellite markers D3Mit106 and D3Mit291 were used to genotype individual DNA samples. The causative mutation was identified by comparing the whole-exome sequences from a homozygous eg/1 mutant and control [25]. Between D3Mit106 and D3Mit291 on Chromosome 3, a unique point mutation c.G344T of Pitx2 was identified in the filtered data of WES (Table S2), in eg/1/eg/1 but not in wild-type DNA. Allele-specific PCR (AS-PCR) was used on genomic DNA to confirm the presence of the Pitx ${ }^{\text {egl1 }}$ mutation. Three oligo primers were selected from exon 2 of the Pitx2 gene with [G/T] single base change $(\mathrm{WT}=\mathrm{G}$, egl1 mutant $=\mathrm{T})$ using a web-based software "Web-based AlelleSpecific Primer" (http://bioinfo.biotec.or.th/WASP): wild-type (W), mutant $(\mathrm{M})$ and common (C) primers: W reverse primer: CGAGTGGACATGTCTGGGTAAC; $M$ reverse primer: CGAGTGGACATGTCTGGGTAAA; $C$ forward primer: AGCAAGGAAAGAATGAGGAT, and the PCR product size $145 \mathrm{bp}$ (base pairs). The AS-PCR assay was conducted in two parallel experiments: (1) "mutant + common" primer experiment (MC) and (2) "wild + common" primer experiment (WC). The PCR assay for Pitx $2^{\text {egli }}$ mutation was performed in $10 \mu \mathrm{l}$ reactions. The PCR conditions were as followed: initial denaturation for $3 \mathrm{~min}$ at $94^{\circ} \mathrm{C}$ followed by 36 cycles of denaturation for $15 \mathrm{~s}$ at $94^{\circ} \mathrm{C}$, annealing for $2 \mathrm{~min}$ at $55^{\circ} \mathrm{C}$, extension for $2 \mathrm{~min}$ at $72{ }^{\circ} \mathrm{C}$, and a final extension step for $7 \mathrm{~min}$ at $72^{\circ} \mathrm{C}$. PCR products were run on $1.5 \%$ SeaKem agarose gel.

\section{Generation of knock-in mouse model by CRISPR/Cas9}

The CRISPR/Cas9 oligonucleotide directed approach was used to generate the Pitx2 p.R115L knock-in (KI) mice. The gRNA (CTTCGCGAGTGGACATGTCTGGG) designed to mouse Pitx2 gene together with Cas9 mRNA and a donor oligo (CAGAGGACTCATTTCACTAGCCAGCAGCTGCAGGAGCTGGAAGCCACTITCCAGAGAAACCTCTATCCAGACATGAGTACTCGCGAAGAAATCGCCGTGTGGACCAACCTTACCGAAGCCCGAGTCCGGGTAGGAGCCAGCACCCAATCTGGGAAAACGAGGGGGCCGAGGCC) containing the R115L (CGC to CTC) mutation were co-injected into C57BL/6J mouse zygotes to generate targeted knock-in offspring. F0 founder animals were mated to C57BL/6J wildtype mice and subject to sequence analysis. Positive F1 offspring (Pitx2 ${ }^{\mathrm{R} 115 \mathrm{~L}}$, strain name C57BL/6J-Pitx $2^{\text {em1ZXJ }}$, hereafter named 
Pitx ${ }^{K l}$ ) were backcrossed to C57BL/6J for four generations and then intercrossed to generate heterozygous and homozygous offspring. After PCR amplification of the targeted region, homozygotes (Pit $\times 2^{K l}$, heterozygotes $\left(\right.$ Pit $\left.\times 2^{K / /+}\right)$, and wild-type (Pitx $2^{W T}$ ) could be determined by Sanger sequencing analysis.

\section{Genotyping of Pitx $2^{K I}$ mice}

Genomic DNA was isolated either from tail tips or from blood using the QIAamp DNA Blood kit (QIAGEN) according to the manufacturer's instructions. WES results or genotypes were confirmed by Sanger sequencing with the following primers (Sangon Biotech, Shanghai, China): Pitx2-Forward primer (F1): 5'-CGGTAGAGAGGTTGTAGATGGGAGTCT-3'; Pitx2-Reverse primer (R1): 5'-GCAGAGAGCCGCTGAGGTTGTAG-3'. All PCR amplification was performed using a master mix (Invitrogen, USA). The first cycle consisted of $95^{\circ} \mathrm{C}$ for $5 \mathrm{~min}$, followed by 32 cycles of $95^{\circ} \mathrm{C}$ for $15 \mathrm{~s}$, $60^{\circ} \mathrm{C}$ for $30 \mathrm{~s}$ and $72^{\circ} \mathrm{C}$ for $30 \mathrm{~s}$. The PCR products were purified by FastAP Thermosensitive Alkaline Phosphatase (Thermo Scientific Fermentas), and directly sequenced using BigDye version 3.1 and an ABI 3730 automated sequencer (Applied Biosystems) according to the manufacturer's instructions.

\section{Plasmids and site-directed mutagenesis}

The recombinant expression plasmids, pcDNA3.1-PITX2-Flag, pcDNA3.1Nrf2-HA tag, and pcDNA3.1-YAP1-HA tag were purchased from Youbio Inc. (Youbio, Changsha, China). Point mutation c. G344T was introduced into the WT Pitx2 cDNA by site-directed mutagenesis using a QuikChange Lightning Site-Directed Mutagenesis Kit (Agilent Technologies, Santa Clara, CA, USA) with a complementary pair of primers: F: $5^{\prime}$-CCACTTTCCAGAGAAACCTCTACCCAGACATG-3' and R: 5'-CATGTCTGGGTAGAGGTTTCTCTGGAAAGTGG-3'. The recombinant plasmids containing PITX2 (R115L)-Flag fusion constructs were sequenced to confirm the desired mutation and to exclude any other sequence variations.

\section{Cell culture and transfection}

HEK293T and COS-7 cells were purchased from National Infrastructure of cell line Resource (Wuhan, China) and were recently authenticated by STR profiling. They were cultured in DMEM with high glucose (Hyclone, South Logan, UT, USA) supplemented with $10 \%$ fetal bovine serum (Gibco, Grand Island, NY, USA) and $100 \mathrm{U} / \mathrm{ml}$ penicillin/streptomycin (Invitrogen, Waltham, MA, USA) in an incubator set to $37^{\circ} \mathrm{C}$ with $5 \% \mathrm{CO}_{2}$. For transfection, cells were seeded in plates (Corning, NY, USA) and transiently transfected with Flag-tagged PITX2 ${ }^{\text {WT }} /$ PITX2 $^{\text {R115L }}$ or HA-tagged NRF2/YAP1 plasmid using Lipofectamine 3000 (Invitrogen, CA, USA) according to the manufacturer's instructions, and the cell lysis were harvested after $48 \mathrm{~h}$.

\section{Immunocytochemistry}

COS-7 cells were seeded in 24-well plates (Corning, Corning, NY, USA) and transfected at $70 \%$ confluency with constructed vectors or empty vectors using Lipofectamine 3000 (Invitrogen, CA, USA) according to the manufacturer's instructions. Cells were harvested after $48 \mathrm{~h}$ and fixed in $4 \%$ paraformaldehyde for $15 \mathrm{~min}$ at room temperature. After blocking with $1 \times$ PBS containing $5 \%$ normal goat serum and $0.2 \%$ Triton X-100, cells were incubated with specific antibodies at $4{ }^{\circ} \mathrm{C}$ overnight. The primary antibodies used are shown in Table S3. AlexaFluor 594/488-conjugated goat anti-mouse/rabbit secondary antibody (Cat\# A11005 and A11008, Invitrogen, Waltham, MA, USA, 1:500 dilution) was applied and nuclei were counter-stained with DAPI (Cat\# D8417, Sigma, St Louis, MO, USA).

\section{Luciferase assay}

The luciferase assays to detect the transcriptional activity of PITX2 were performed as described previously $[26,27]$. The human LEF-1 promoters were constructed in the luciferase vector as previously described $[28,29]$. SV-40 $\beta$-galactosidase reporter plasmid was used as a control for transfection efficiency. 293T cells were prepared and mixed with $2.5 \mu \mathrm{g}$ of expression plasmids, $5 \mu \mathrm{g}$ of reporter plasmid and $0.5 \mu \mathrm{g}$ of SV- 40 $\beta$-galactosidase plasmid in $60 \mathrm{~mm}$ culture dishes. After incubating for $48 \mathrm{~h}$, transfected cells were lysed and assayed for reporter activities. Luciferase activity was measured using reagents from Promega (Cat\# E1500, Madison, WI, USA). $\beta$-galactosidase was measured using Galacto-Plus reagents (Cat\# T2118, Invitrogen, Waltham, MA, USA). All luciferase activities were normalized to $\beta$-galactosidase activity and are shown as mean-fold differences relative to empty luciferase plasmids.

\section{RNA-sequencing analysis}

RNA-sequencing analysis was performed on four independent biological replicates from four wild-type (WT) and four Pitx2 mutant retina at 2 months of age. After harvesting, both retinas for each animal were collected and immediately frozen. RNA was extracted using RNeasy Mini kits (QIAGEN). RNA integrity and concentration were evaluated using a BioAnalyzer 2100 with RNA 6000 Nano Kit (Agilent Technologies, Santa Clara, CA, USA). For library preparation, a total of $2 \mu \mathrm{g}$ RNA per sample was used as input material for the RNA sample preparations. Sequencing libraries were generated using NEB Next, Ultra RNA Library Prep Kit for Illumina ${ }^{\circledR}$ (Cat\# E7530L, NEB, USA), following the manufacturer's recommendations, and index codes were added to attribute sequences to each sample. Briefly, mRNA was purified from total RNA using poly-T oligoattached magnetic beads. Fragmentation was carried out using divalent cations under elevated temperature conditions in NEBNext First-Strand Synthesis Reaction Buffer ( $5 \times)$. First-strand cDNA was synthesized using random hexamer primers and RNase $\mathrm{H}$. Second-strand $\mathrm{CDNA}$ synthesis was subsequently performed using dNTPs, DNA polymerase I and RNase $\mathrm{H}$. The library fragments were purified with QiaQuick PCR kits and eluted with EB buffer, and then terminal repair, A-tailing and addition of an adapter were implemented. The RNA concentration of the library was measured using a Qubit RNA Assay Kit in Qubit 3.0 and the library was diluted to $1 \mathrm{ng} / \mu \mathrm{l}$. Insert sizes were assessed using the Agilent Bioanalyzer 2100 system (Agilent Technologies), and the qualified insert size was accurately quantified using a StepOnePlus ${ }^{T M}$ Real-Time PCR System (library valid concentration $>10 \mathrm{nM}$ ). The clustering of the index-coded samples was performed on a cBot cluster generation system using a HiSeq PE Cluster Kit v4-cBot-HS (Illumina, CA, USA) according to the manufacturer's instructions. After cluster generation, the libraries were sequenced on an Illumina HiSeq 2500 platform, and $150 \mathrm{bp}$ paired-end reads were generated. All gene expression values from RNA-seq were converted to a log2 value and analyzed further. Then, a $P$ value of less than or equal to 0.05 was considered significant. The raw sequence data have been deposited in the Genome Sequence Archive (Genomics, Proteomics \& Bioinformatics 2021) in National Genomics Data Center, Beijing Institute of Genomics, Chinese Academy of Sciences, under accession number CRA005112 that are publicly accessible at "https://ngdc.cncb.ac.cn/gsa".

\section{RNA extraction and quantitative PCR}

Optic nerve total RNA was extracted using TRlzol reagent (Sigma, Saint Louis, MO, USA) as recommended by the manufacturer. First-strand CDNA was synthesized using iScript cDNA Synthesis Kit (Bio-Rad, Hercules, California, USA). Quantitative PCR was carried out using iTaq SYBRMix (BioRad, Hercules, California, USA) and a CFX384 Touch Real-Time PCR Detection System (Bio-Rad, Hercules, California, USA). Primers were designed using Primer3Plus or from published resources. Table S4 shows the specific primer sequences used. Four biological replicates were used for each sample. All target genes were normalized to actin mRNA levels and fold change were calculated by performing delta-delta $\mathrm{Ct}$ analysis [30].

\section{Histological analysis}

For haematoxylin and eosin staining (H\&E), enucleated eyes from control and mutant mice were fixed overnight in $1.22 \%$ glutaraldehyde and $0.8 \%$ paraformaldehyde in $0.08 \mathrm{M}$ phosphate buffer, embedded in paraffin and then cut in $5 \mu \mathrm{m}$ sections. To ensure sections used for quantification came from the same eccentricity, the globe was embedded in the same orientation. Sections that encompassed the optic nerve (ON) were selected for staining with haematoxylin and eosin according to standard protocol.

\section{Immunohistochemistry}

For immunohistochemistry, eyes were removed from euthanized mice by intraperitoneal injection of pentobarbital $(75 \mathrm{mg} / \mathrm{kg})$, and by cervical dislocation and fixed in $4 \%$ paraformaldehyde in $100 \mathrm{mM}$ phosphate buffer $(\mathrm{pH} 7.4)$ for $1 \mathrm{~h}$ at $4{ }^{\circ} \mathrm{C}$, followed by cryoprotection in $30 \%$ sucrose for $2 \mathrm{~h}$. Lens were removed and eyes were embedded in optimal cutting temperature solution (OCT) and sectioned at $10 \mu \mathrm{m}$ thickness. After blocking and permeabilization with $10 \%$ normal donkey serum and $0.2 \%$ Triton X-100 in phosphate buffer for $1 \mathrm{~h}$, the sections were labeled with the primary antibody at $4{ }^{\circ} \mathrm{C}$ overnight. The primary antibodies used are shown in Table S3. The sections were rinsed in PBS three times and Alexa Fluor 594/488-conjugated goat anti-mouse/rabbit secondary antibody (Cat\# A11005 and A11008, Invitrogen, Waltham, MA, USA, 1:500 dilution) was applied and nuclei were counter-stained with DAPI (Cat\# D8417, Sigma, St 
Louis, MO, USA) for $1 \mathrm{~h}$ at room temperature. Images were captured on a Zeiss LSM 800 confocal scanning microscope. In addition, the expression levels of CD68 and GFAP were compared between WT and mutant retinas by quantifying their fluorescence intensities using ImageJ software.

\section{Retinal flat mounts and RGC count}

Retinas were dissected from enucleated eyes and flattened. Retinas were immersed in $4 \%$ PFA for $24 \mathrm{~h}$ at $4{ }^{\circ} \mathrm{C}$, then blocked in PBS containing $1 \%$ bovine serum albumin and $0.5 \%$ Triton X-100 and incubated with polyclonal rabbit anti-Brn3a antibody (Cat\#ab245230, Abcam, Cambridge, USA, 1:200 dilution) for $12 \mathrm{~h}$ at $4{ }^{\circ} \mathrm{C}$. After several washes, the retinas were incubated with the secondary antibody, AlexaFluor 488-conjugated goat anti-rabbit secondary antibody (Cat\#A11008, Invitrogen, Waltham, MA, USA, 1:250 dilution) for $4 \mathrm{~h}$ at room temperature, and subsequently washed with PBS. To precisely quantitate the loss of RGCs, each retinal quadrant was divided into four zones-nasal, temporal, superior, and inferior-each located $1 \mathrm{~mm}$ from the optic nerve head. RGCs were counted in each unit area by two investigators in a blinded fashion, and the scores were averaged.

\section{Semithin-section processing of the optic nerve}

Optic nerves were dissected from eyes and placed in a solution containing $4 \%$ paraformaldehyde and $0.2 \%$ glutaraldehyde for $24 \mathrm{~h}$. After rinsing three times in $0.1 \mathrm{M}$ phosphate buffer $(\mathrm{pH} 7.4)$ for $15 \mathrm{~min}$ each, tissues were fix with $1 \%$ osmic acid and $0.1 \mathrm{M}$ phosphate buffer $(\mathrm{pH} 7.4)$ at room temperature $\left(20^{\circ} \mathrm{C}\right)$ for $2 \mathrm{~h}$. Osmic acid fixed tissues were rinsed three times in $0.1 \mathrm{M}$ phosphate buffer ( $\mathrm{pH} 7.4$ ) for $15 \mathrm{~min}$ each, and dehydrated in graded concentrations of ethanol. The dehydrated tissues were embedded in acetone: 812 embedding medium $=1: 1$ for $2-4 \mathrm{~h}$, and in acetone: 812 embedding medium $=1: 2$ infiltration, overnight, and finally in 812 embedding medium for 5-8 h. Samples were inserted into an embedding plate containing 812 embedding medium, and placed overnight in an incubator at $37^{\circ} \mathrm{C}$, followed by polymerization at $60^{\circ} \mathrm{C}$ for $72 \mathrm{~h}$. Semithin sections $(1 \mu \mathrm{m})$ were cut transversely from segments of optic nerves caudal to the eyeball and stained with $1 \%$ toluidine blue.

\section{TUNEL assay}

Apoptotic cell death was detected in prepared frozen sections by the terminal deoxynucleotidyl transferase-mediated biotinylated UTP nick end labeling (TUNEL) assay according to the manufacturer's protocol (Cat\#11684795910, Roche Diagnostics, Indianapolis, IN). Images were captured on a Zeiss LSM 800 confocal scanning microscope.

\section{Immunoblotting}

Samples for western analysis were lysed in standard RIPA lysis buffer $(50 \mathrm{mM}$ Tris- $\mathrm{HCl}, 150 \mathrm{mM} \mathrm{NaCl}, 1 \%$ Triton $\mathrm{X}-100,0.5 \%$ sodium deoxycholate, $0.1 \%$ SDS, pH 7.4) supplemented with Complete Protease Inhibitor Cocktail (Roche). The protein content of the samples were quantified by a standard BCA method. SDS-PAGE was performed using standard techniques. Blots were blocked with $8 \%$ non-fat dry milk in TBST for $2 \mathrm{~h}$ at room temperature and subsequently incubated with primary antibodies in blocking solution overnight at $4^{\circ} \mathrm{C}$. The primary antibodies used were shown in Table S3. Primary antibodies were detected with either an antimouse or anti-rabbit HRP-conjugated secondary antibody (1:5000; BioRad), and signals were developed using SuperSignal West Pico Chemiluminescent Substrate (Pierce). ImageJ was used to calculate the relative density of the protein. At least three independent western blots were conducted, and one representative blot is presented.

\section{Coimmunoprecipitation and quantification}

Transfected 293T cells were harvested and lysed in lysis buffer $(50 \mathrm{mM}$ Tris$\mathrm{HCl}, \mathrm{pH} 7.5,150 \mathrm{mM} \mathrm{NaCl}, 1 \%$ Nonidet P-40, $0.5 \%$ sodium deoxycholate and $1 \%$ protease inhibitor cocktails; Sigma, St. Louis, MO, USA) and incubated on ice for $5 \mathrm{~min}$ with periodic agitation. Cell lysates were centrifuged, and the supernatant was incubated with antibodies of interest and Protein G Plus/Protein A agarose beads (Sigma, St. Louis, MO, USA) or Flag M2-conjugated agarose beads (Sigma, St. Louis, MO, USA) at $4{ }^{\circ} \mathrm{C}$ overnight. The beads were washed six times with cell lysis buffer, and the precipitated proteins were further analyzed by western analysis.

To quantify the interaction between WT/mutant PITX2 and NRF2/ YAP1, ImageJ was used to calculate the intensity of the targeted protein. The intensity of IP bands/ input bands was calculated to normalize the total protein content. The relative binding capacity was quantified by the normalized IP-HA intensity/normalized IP-Flag intensity. At least three independent co-IP experiments were conducted, and one typical blot is presented.

\section{Measurement of superoxide production}

Superoxide production was evaluated in retinal cryosections using dihydroethidium (DHE) as described previously [31]. Briefly, frozen sections were incubated with DHE $(2 \mu \mathrm{M})$ for $30 \mathrm{~min}$ at $37^{\circ} \mathrm{C}$. DHE is oxidized upon reaction with superoxide to ethidium bromide, which binds to DNA in the nucleus and fluoresces red. Excessive reactive oxygen species (ROS) production is a hallmark of oxidative stress. Thus, retinal levels of superoxide, as determined by DHE staining with subsequent quantification of fluorescence intensity, was measured. Images were captured on a Zeiss LSM 800 confocal scanning microscope. The relative fluorescence intensity within the images obtained was determined via automated image analysis of ZEISS ZEN Intellesis or ImageJ software.

\section{Measurement of the MDA level and SOD, GSH-Px activity}

Retinas were immediately extracted from enucleated eyes. The weighed retinal samples were prepared as a $10 \%$ homogenate in $0.9 \%$ saline. After homogenization on ice, the homogenate was sedimented at $2000 \times g$ for $10 \mathrm{~min}$, and the supernatant was collected and diluted. The GSH-Px, SOD activity, and MDA levels of the retinal lysates were determined using ELISA kits from the Nanjing Jiancheng Bio-company (Cat\#A005, A001, A003, respectively, Nanjing, China). All procedures were carried out according to the manufacturer's instructions.

\section{Statistical analysis}

Statistical analysis was performed using GraphPad Prism 6 software. The data sets were tested for normal distribution using Shapiro-Wilk test. For normally distributed data, statistical significance was determined by Student's $t$-test or ANOVA. If the data was not normally distributed, nonparametric statistic was used. $p$-values were calculated by Student's $t$-test or ANOVA followed by a, Tukey, Dunnett or Sidak's multiple comparisons test as appropriate. $P<0.05$ was considered statistically significant.

\section{RESULTS}

\section{A new model of early-onset glaucoma identified in an ENU} screening program

The eg/1 mutant, with bulging eyes, was discovered while screening an ENU treated cohort for craniofacial phenotypes. Affected mice were distinguished from $\mathrm{C} 57 \mathrm{BL} / 6 \mathrm{~J}$ controls by a high IOP phenotype (Fig. 1A). Optic neuropathy characterized by optic nerve cupping, and by severe retinal nerve fiber layer (NFL) loss in mutant retinas (Fig. 1B) was detected with optical coherence tomography. Histological study of retinas from 12month-old mice confirmed the clinical assessment, and revealed enlarged optic disc cup with thinning of the inner retinal layer in mutant mice (Fig. 1C). Whole-exome sequences (WES) revealed a $\mathrm{G}$ to $\mathrm{T}$ base-pair transversion in Pitx2 (Fig. 2A-D). Allele-specific PCR (Fig. S1) and Sanger sequencing (Fig. S2) further confirmed the presence of Pitx $2^{\text {egl1 }}$ mutation in a segregating cross. Thus, a novel homozygous missense mutation, c. G344T, p. R115L (NM_001042502.2), in the Pitx2 gene was identified and deemed the most likely candidate for the glaucoma-like symptom in egl1/ eg/1 mice. Notably, the affected amino acid residue is located in the HD region of PITX2 protein (Fig. 2D, lower panel) and is highly conserved across species (Fig. 2E).

\section{Generation of the Pitx $2^{\text {Mut }}$ mouse model}

In order to confirm the causative nature of the eg/1 mutation and explore the Pitx2 mutation further, a Pitx $2^{R 115 L}$ knock-in mouse model (named $\mathrm{KI}$ ) was generated using CRISPR/Cas9 technology (Fig. S3). Homozygous Pitx $2^{K I}$ mice were born at the expected Mendelian ratio (47 out of 220 in Pitx $2^{K I /+}$ to Pitx $2^{K I /+}$ crosses) and was not significantly different from the expected, 55 out of 220 mice. No significant changes in the protein content and localization pattern of PITX2 were observed in mutant retina 

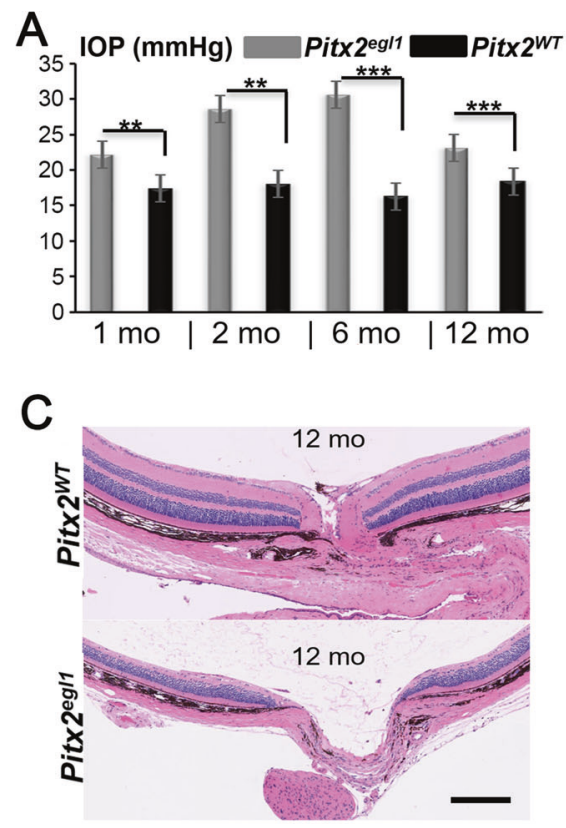

Fig. 1 Pitx $2^{\text {eg/1 }}$ mice exhibit an early-onset glaucoma phenotype. A Longitudinal intraocular pressure analysis of Pitx $2^{W T}$ and Pitx $2^{\text {egl1 }}$ mice ( $n=12$ for each group). Intraocular pressure (IOP) measurements with a rebound tonometer (TonoLab) showed a progressive increase in pressure in Pitx ${ }^{\text {egl1 }}$ mutant mice. IOP (gray bar, egl1 mice $=22.1 \pm 3.4$ and black bar, WT mice $=17.4 \pm 2.2$ ) at 1 month of age. IOP (gray bar, egl1 mice $=28.6 \pm 5.0$ and black bar, WT mice $=18.0 \pm 1.7$ ) at 2 months of age. IOP (gray bar, eg/1 mice $=30.6 \pm 6.5$ and black bar, WT mice $=16.3 \pm$ 1.3) at 6 months of age. IOP (gray bar, egl1 mice $=23.1 \pm 1.4$ and black bar, WT mice $=18.4 \pm 1.7$ ) at 12 months of age. B Representative fundus photographs and optical coherence tomography images of Pitx $2^{W T}$ and Pitx $2^{\text {egli }}$ mice at different ages. A partial ring around optic cup in the fundus and and optic nerve cupping observed by OCT in Pitx ${ }^{\text {egl/ }}$ mice (bottom row), but not in Pitx ${ }^{\text {WT }}$ mice (upper rows) at 2, 6, and 12 months of age. The green lines indicate the plane in which the B-scan to the left of the fundus images were taken $\mathbf{C}$ Representative images of histologic sections taken through nerve heads from Pitx $2^{W T}$ and Pitx $2^{\text {egli }}$ mice at 12 months of age. Optic nerve head cupping was observed in Pitx ${ }^{\text {eg/l }}$ mice, with thinning of inner retina layers. Scale bar: $200 \mu \mathrm{m}$. ${ }^{* *} P<0.01 ;{ }^{* * *} P<0.001$. Data are presented as the mean \pm SEM.

compared to that of control (Fig. S3C, D). Immunofluorescent staining of sections from WT mice indicated that PITX2 was strongly expressed in the mouse $\mathrm{GCL}$, where it colocalized with the RGC marker, Brn3a (Fig. S3D, upper panel), suggesting that PITX2 may play a role in RGC function.

\section{Pitx2 ${ }^{K I}$ mice develop elevated IOP subsequent to anterior segment dysgenesis}

Similar to the Pit $x 2^{\text {egl1 }}$ mutant mice, we observed that approximately $59 \%$ of mutants (20 out of 34 ) exhibited a bulging and distended appearance of the eye with a mild corneal opacity (Fig. 3A), as early as 2 months of age. Moreover that roughly $80 \%$ of these cases were bilaterally affected, as reported for ARS patients [19]. Rebound tonometry confirmed elevated IOPs in mutants. As expected, Pitx $2^{K I}$ mice exhibited a progressive elevation of IOP measurements, $13.87 \pm 0.89 \mathrm{mmHg}(n=16)$, $16.38 \pm 1.13 \mathrm{mmHg}(n=16)$, and $18.78 \pm 1.54 \mathrm{mmHg}(n=18)$ at 3,6 weeks, and 4 months of age, respectively. In contrast, the IOP measuring of their control littermates at corresponding ages were $11.16 \pm 1.58 \mathrm{mmHg} \quad(n=16), 13.17 \pm 1.88 \mathrm{mmHg} \quad(n=16)$, and $14.62 \pm 2.34 \mathrm{mmHg}(n=18)$ (Fig. 3B), which is consistent with previous measurements for C57BL/6Jstrains [32]. By 4-months of age, $47 \%$ of mutants presented with high IOP levels of $>18 \mathrm{mmHg}$, and $\sim 14 \%$ mutants with IOP $>21 \mathrm{mmHg}$, a level commonly associated with glaucoma (Fig. 3C). Interestingly, nearly $53 \%$ of mutants did not develop ocular hypertension at this stage indicating incomplete penetrance of the disease. Similarly, only $50 \%$ of ARS patients develop elevated IOP and glaucomatous pathologies [33-35].

Previous studies have demonstrated that PITX2 is required for normal ocular development $[21,36]$. We, therefore, sought to determine whether the elevated IOP in Pixt $2^{K I}$ mice was associated with aberrant ocular development. Mice were examined at E15.5,
P9 and 6 weeks for morphological alterations by histology. The anterior chamber of mutant mice at E15.5 appeared slightly collapsed and showed delayed separation of the cornea from the lens surface compared to Pitx $2^{\text {Wt }}$ mice, but otherwise no other suggestion of abnormal development was apparent (Fig. 3D, upper panel). By contrast at P9, multiple malformations of the anterior chamber were observed in mutant eyes. A distinct disruption of the iridocorneal angle with adhesion of the iris to cornea, creating a fully angle closure was observed in mutant eyes (Fig. 3D, middle panel). The iris-cornea adhesion was more pronounced in adults, at 7 weeks of age, and was accompanied by iris hypoplasia and ciliary body atrophy in all mutant eyes (Fig. 3D, lower panel).

Moreover, histological analysis in 7-week-old Pitx2 mutant mice revealed a thinner cornea compared to that of control, with a reduced thickness of both the epithelial and stromal layers (Fig. 3D). Detailed longitudinal analysis of the mutant and WT mice from P16, 6-week- to 3-month-of age confirmed the reduced central corneal thickness (Fig. 3E, F), suggesting an abnormal corneal development. In addition, the TM and Schlemm's canal (SC) in 1-month-old mutants were occluded and not readily identifiable, and the trabecular endothelial cells were tapered and attenuated without regular cellular components (Fig. 3G, upper panel). To further examine the TM malformation, ocular frozen sections were stained for a-SMA, a marker for TM cells. This analysis revealed that the majority of mutants exhibited an absence or smaller area of a-SMA staining compare with that of control, corroborating the TM hypoplasia in Pitx $2^{K l}$ mice (Fig. 3G, $\mathrm{H})$. It should be noted that all heterozygotes (Pit $x 2^{K I /+}$ ) tested at 3 months of age were not significantly different in ocular morphology or in IOP levels compared to their WT littermates (Fig. S4A, B), and all retinal structures, including the optic nerve head and anterior segment appeared normal (Fig. S4B, C). 
A Linkage cross data

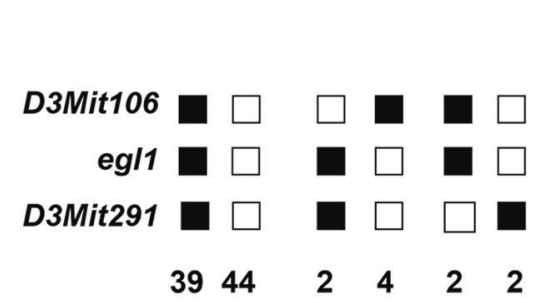

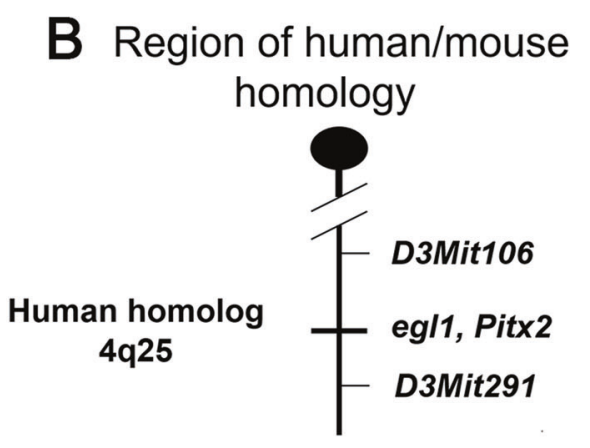

C IGV on Pitx $2^{\text {egli }}$ genome sequence (exon 2)

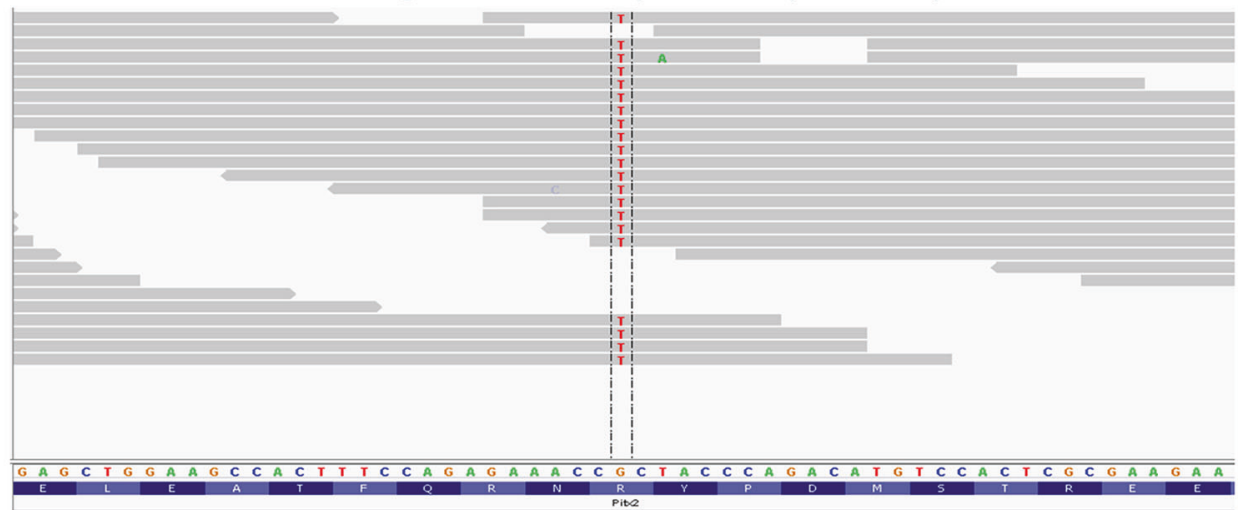

D

E

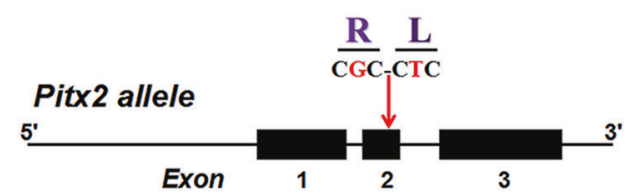

Pitx2 protein

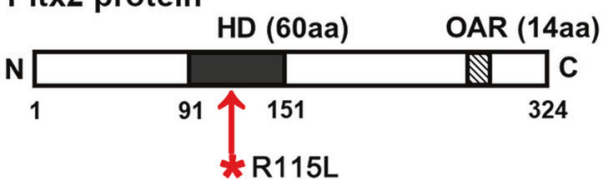

Organism

M.musculus

H.sapiens

P.troglodytes

M.mulatta

C.Iupus

B.taurus

R.norvegicus

G.gallus

D.rerio

D.melanogaster

\section{A.gambiae \\ $X$.tropicalis}

p.R115L

RNRYPDMSTR

RNRYPDMSTR

RNRYPDMSTR

RNRYPDMSTR

RNRYPDMSTR

RNRYPDMSTR

RNRYPDMSTR

RNRYPDMSTR

RNRYPDMSTR

RNRYPDMSTR

RNRYPDMSTR

RNRYPDMSTR

Fig. 2 Identification of Pitx2 mutation in Pitx2 ${ }^{\text {egl1 }}$ mice by whole-exome sequencing. A Genetic and molecular analysis of the eg/1 strain. A total of 93 progeny from a eg/1/eg/1 X (eg/1/DBA/2J) F1 backcross were genotyped in a genome-wide scan. Linkage to several markers on mouse Chr 3 was observed. The columns of squares represent haplotypes (filled boxes, eg/1/eg/1 B6 alleles; open boxes, eg/1B6/DBA/2J allele). The number of chromosomes associated with each haplotype is indicated below each column. B Genetic map of Chr 3 in the eg/1 region showing the closest markers and the region of human homology. C Integrative Genomics Viewer (IGV) shows the base substitution. D A homozygous missense mutation, c. G344T, p. R115L of the Pitx2 gene was identified in Pitx $2^{\text {egll }}$ mice by whole-exome sequencing (WES). Upper panel: The schematic diagram shows the location of the missense mutation in the Pitx2 gene. Bottom panel: Functional domains of PITX2 protein with the location of the mutations. HD, homeodomain; OAR, opt aristaless and rax. E Protein sequence alignment of amino sequences surrounding the Pitx2 mutation with orthologous sequences from Mus musculus to Xenopus tropicalis. The mutant amino acid residue is in red. Note that the amino acid sequences surrounding the affected amino acid residues are also highly conserved.

\section{Retinal ganglion cell loss and optic nerve degeneration in} Pitx2 ${ }^{K I}$ mice

To determine whether high IOP leads to optic neuropathy in Pitx $2^{K I}$ eyes, we assessed retinal and optic nerve morphology in histological sections ON. Eyes of P16 mutant mice, an age prior to IOP elevation, exhibited a normal retinal layer morphology
(Fig. S5A). At 6 weeks, a mild loss of cells in the ganglion cell layer (GCL) was observed in mutant mice (Fig. S5B), and this trend generally progressed with age.

At 3 months, mutant mice showed a mild thinning of outer and inner nuclear layers (Fig. S6A, B), accompanied by a $61.2 \%$ decrease in GCL cell number compared to that of controls 
A

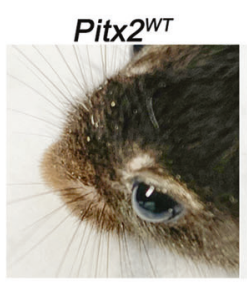

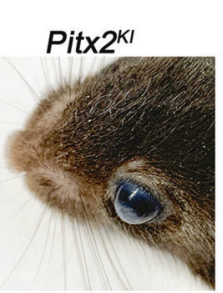

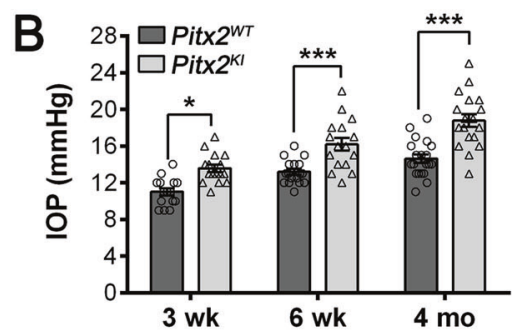

C
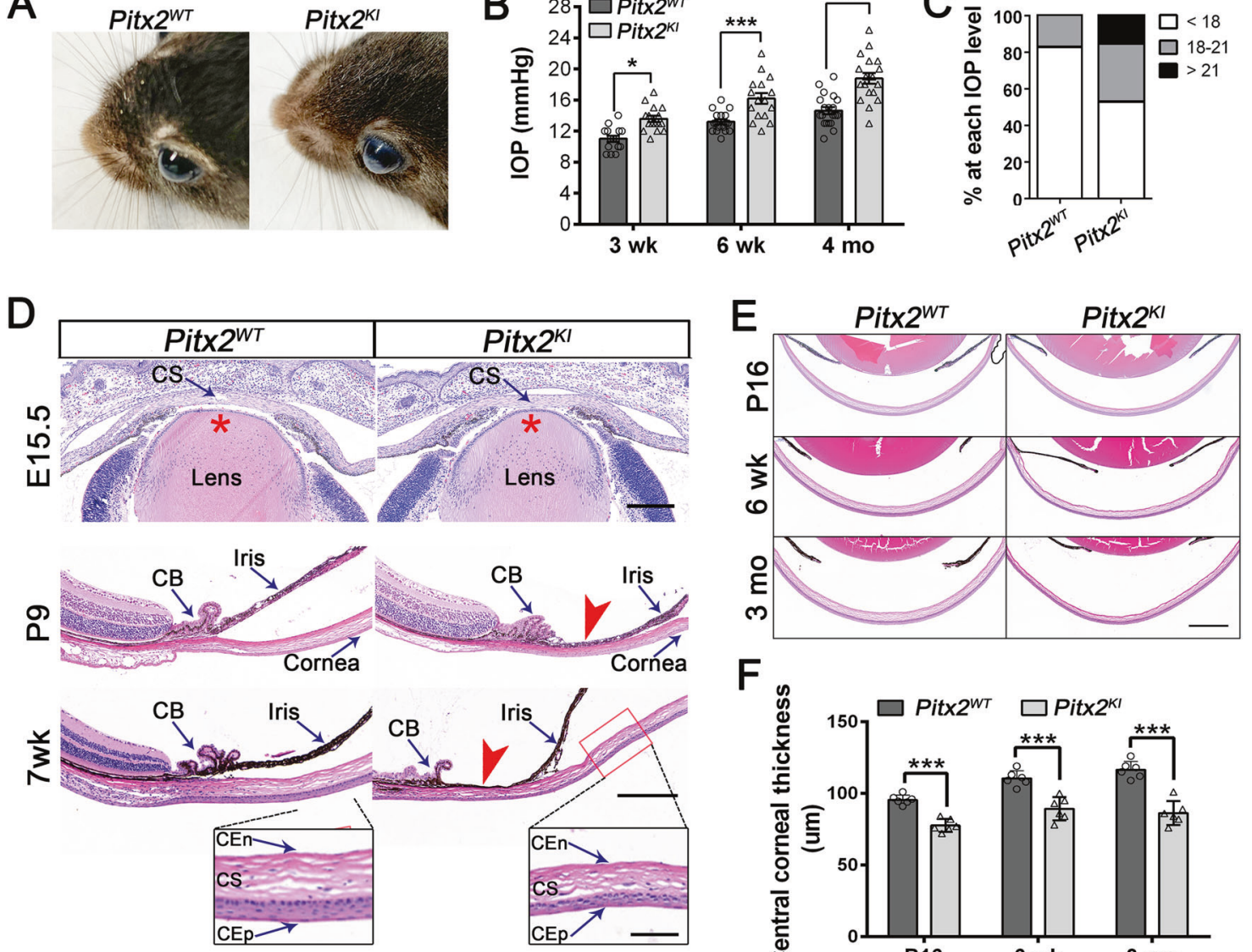

$\mathbf{F}$
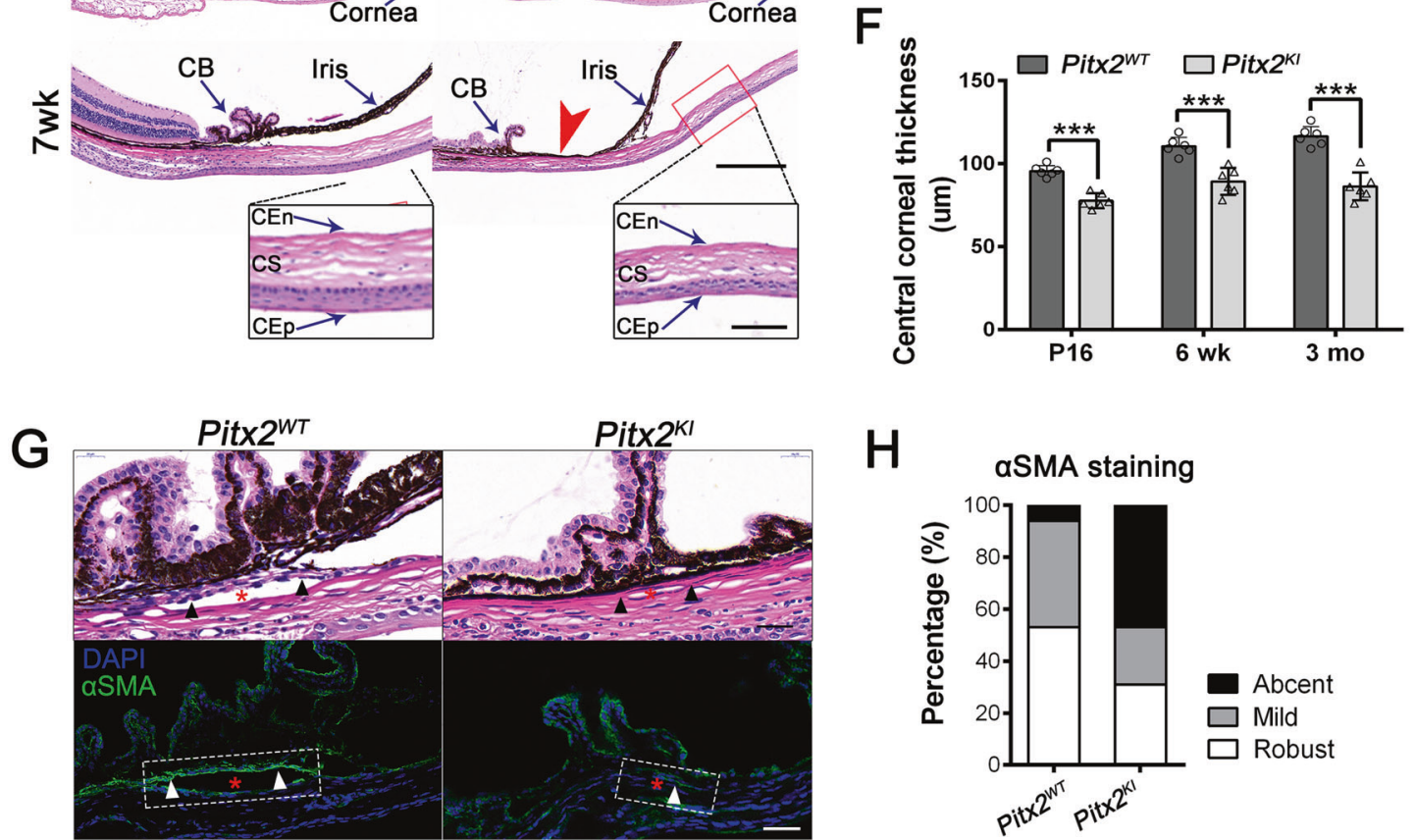

$\mathrm{H}$

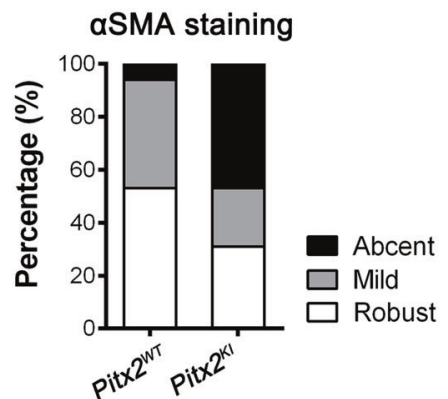

Fig. 3 Elevated intraocular pressure and hypoplasia of ocular anterior segment in Pitx $2^{K I}$ mice. A Image of Pitx ${ }^{W T}$ and Pitx $2^{K l}$ adult mice at 4 months of age. B Intraocular pressure analysis in the Pitx $2^{W T}$ and Pitx $2^{K I}$ mice at 3 weeks $(n=16), 6$ weeks $(n=16)$ and 4 months $(n=18)$ of age. Individual IOP values were shown as dots in the histogram. C Histogram showing the proportion of eyes in each IOP range at 4 months. D Hematoxylin and eosin (H\&E) staining of eyes of Pitx $2^{W T}$ and Pitx $2^{K I}$ mice at E15.5, P9, and 7 weeks. Compared with Pitx $2^{W T}$ mice, anteriorsegment development in E15.5 Pitx $2^{K T}$ mice was slightly impaired. Scale bars: $100 \mu \mathrm{m}$. Iris-cornea adhesion and closed iridocorneal angle (red arrows) were detected in mutant mice at P9 and 7 weeks of age. Scale bars: $200 \mu \mathrm{m}$. The bottom panels show high-magnification images of the boxed areas, which denotes a thinner corneal epithelium in Pitx ${ }^{K I}$ mice at 7 weeks. Scale bars: $50 \mu \mathrm{m}$. CB, ciliary body; CEn, corneal endothelium; CEp, corneal epithelium; CS, corneal stroma. E H\&E-stained sagittal sections of E15.5, P9, and 7 weeks old WT littermates and mutant samples. Scale bars: $200 \mu \mathrm{m}$. F A comparison of central corneal thickness between mutants and their WT littermates $(n=6$ in each group). Mutant mice present a thinner central cornea relative to their WT littermates. Individual thickness values are shown by dots in the histograms. ${ }^{*} P<0.05 ;{ }^{* *} P<0.001$. Data are presented as mean \pm SEM. G Position-matched images of the H\&E staining (upper panel) and the immunofluorescent staining (lower panel) of optic sagittal sections are shown. Trabecular meshwork (TM) is labeled with alpha-smooth muscle actin ( $\alpha$-SMA), and nuclei counter-stained with DAPI. Conventional outflow tissues are outlined by white boxes. Asterisks indicate Schlemm's canal (SC) lumen, arrowheads show trabecular meshwork. Scale bars: $25 \mu \mathrm{m}$. $\mathbf{H}$ Data show the percentage of $\alpha$-SMA staining measurements in the boxed TM region. Staining was assessed by ratings of "robust", "mild", or "absent". ( $n=6$ in each group). * $P<0.05$; *** $P<$ 0.001. Data are presented as mean \pm SEM.

(Fig. S6A, C), indicating significant RGC loss. This result was precisely confirmed with anti-Brn3a, an RGC-specific marker, stained retinal section from 6-week-old (Fig. 4A) and 3-monthold (Fig. 4B) mice, which demonstrated a progressive RGC death in mutants (Fig. 4C). RGC density was further examined with antiBrn3a stained retinal flatmounts from 3-month-old animals. Each anti-Brn3a stained retinal flatmount was segmented into nasal, temporal, ventral, and dorsal quadrants, and RGC density 

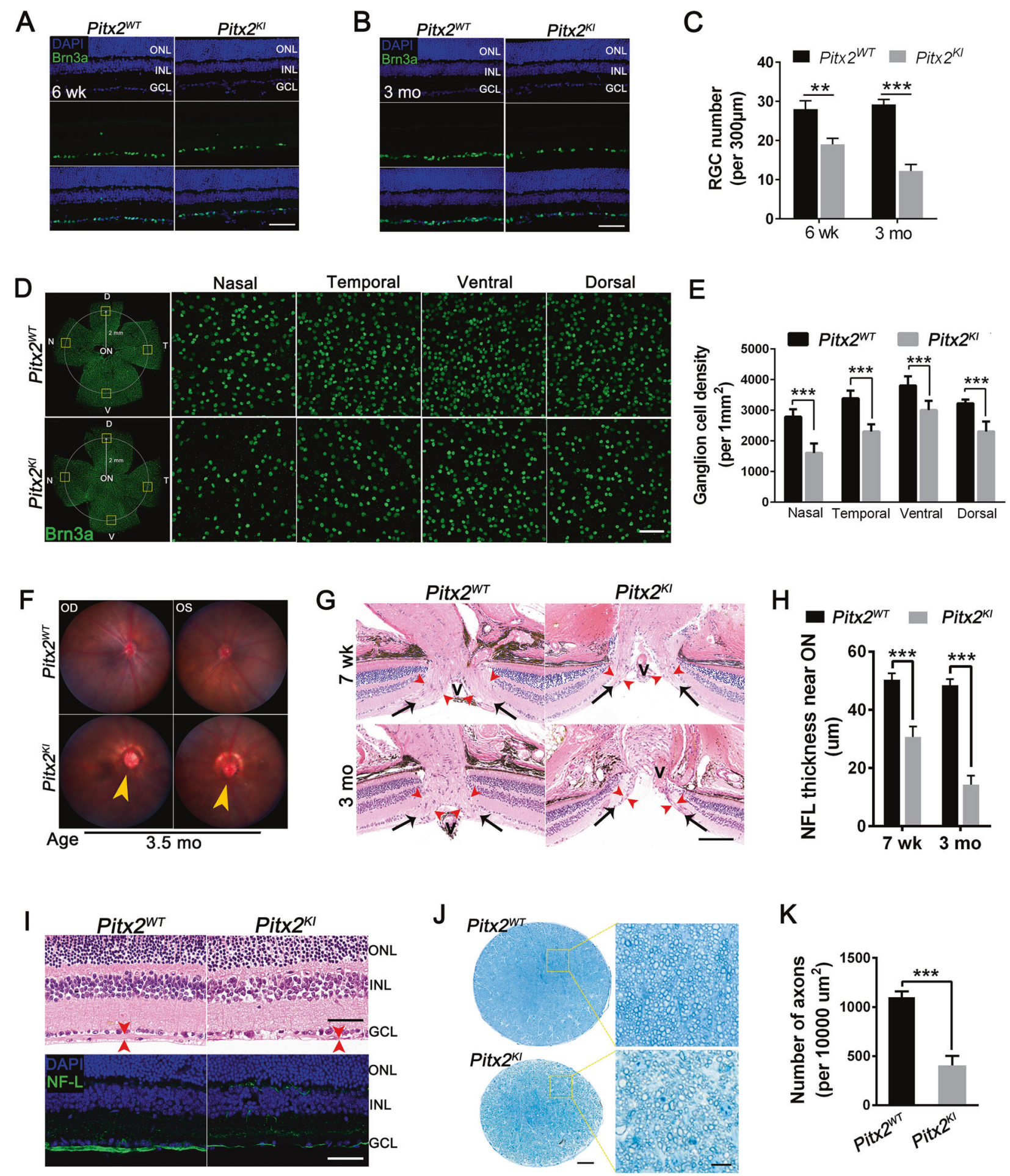

quantified respectively. In mutant retinas, a significant decrease in Brn3a-positive cells in all four quadrants was observed (Fig. 4D). Quantitative analysis revealed a $43.4 \% ; 32.8 \% ; 24.3 \%$, and $27.5 \%$ reduction of Brn3a-positive cells compare to that of WT in nasal, temporal, ventral, and dorsal area, respectively (Fig. 4E).

We next evaluated optic disc cupping, a characteristic pathology of glaucoma. Clinical fundus examination revealed a glaucomatous fundus appearance with an asymmetric and severely excavated $\mathrm{ON}$ head and peripapillary chorioretinal atrophy in mutant eyes (Fig. 4F). Consistent with clinical fundus examinations, histological analysis revealed optic disc cupping and thinning of the NFL near the ON in mutant mice at 7 weeks of age, and these phenotypes became more severe at 3 months (Fig. $4 G, H)$. Moreover, severe axon loss was also visible in the central retina of mutant mice, as evidenced by an extremely atrophied $N F L$, which was confirmed by Neurofilament-L staining (Fig. 4l). To determine details of axonopathy, toluidine blue-stained semithin sections of the ON from 3-month-old animals were prepared and the number of axons were quantified. Compared with WT mice, the axons density was clearly lower in mutant mice and was 
Fig. 4 Ganglion cell loss and optic nerve atrophy in Pitx $2^{K I}$ mutant mice. A, B Retinal RGCs from 6-week-old (A) and 3-month-old (B) mice were labeled with anti-Brn3a, and the nuclei were counter-stained with DAPI. Scale bars: $25 \mu \mathrm{m}$. C Quantification of the Brn3a ${ }^{+}$RGC number in GCL. Pitx $2^{K l}$ mutant mice at 3 months of age exhibit slightly thinner inner and outer nuclear layers compared with WT mice ( $n=6$ per cohort). D Representative immunofluorescent enface images of enface view of a retinal flatmount from WT and mutants at 3 months of age labeled with anti-Brn3a for detecting RGCs. Scale bars: $50 \mu \mathrm{m}$. E Pitx $2^{K l}$ mutants have significantly decreased numbers of RGCs per mm ${ }^{2}(n=8)$ relative to their control littermates $(n=10)$. F Fundus photograph at 5 months shows abnormal morphology of optic nerve head (arrowhead) in the eyes of Pitx $2^{K I}$ mutant mice. G Representative images of histologic sections taken through nerve heads from Pitx $2^{W T}$ and Pitx $2^{K I}$ mice at 7 weeks and 3 months of age. The optic nerve head appeared abnormal in Pitx $2^{K l}$ mice at both ages, with thinning of the nerve fiber layer (NFL) (red arrowheads) and optic nerve excavation is noted (asterisk). Black arrows indicate NFL entering the optic nerve (ON); V: Central blood vessel. Scale bars: $200 \mu \mathrm{m}$. H Quantitation and comparison of the NFL thickness near the optic nerve in 7-week and 3-month-old mice $(n=6$ per cohort). I Position-matched images of the H\&E staining (upper panel) and the immunofluorescent staining (lower panel) of retinal cross sections are shown. Retinal nerve fibers were labeled with neurofilament-L (NF-L), and the nuclei were counter-stained with DAPI. Scale bars: $25 \mu \mathrm{m}$. J Optic nerve atrophy was assessed in cross semithin sections of resin-embedded optic nerves from 5-month-old WT and mutant mice stained with toluidine blue staining. Scale bars: $100 \mu \mathrm{m}$. The panels on the right show high-magnification images of the boxed areas shown in the panels on the left. Scale bars: $10 \mu \mathrm{m}$. K Quantification of axon number in the optic nerve of 5-month-old mice ( $n=10$ per cohort). The Pitx $2^{K l}$ mutants have significantly less axonal projections compared to Pitx ${ }^{W T}$ mice. ONL, outer nuclear layer; INL, inner nuclear layer; $\mathrm{GCL}$, ganglion cell layer. The statistics were calculated based on data from all tested mutants. The representative images of Pitx $2^{K l}$ were from mutants with elevated IOPs. ${ }^{* *} P<0.01 ;{ }^{* *} P<0.001$. \#, no significant difference. The data are presented as mean \pm SEM.

accompanied by axonal swelling and extensive myelin debris (Fig. 4J, K), indicating severe axonal damage. Additionally, to assess if pathological changes in mutants with normal IOPs $(\leq 18)$ occurred, we also examined these mutants. Interestingly, pathologic features of glaucomatous injuries, such as RGC loss (Fig. S7A, B) and ON damage (Fig. S7C-E). were observed but the phenotypes appeared milder compared to mutants with elevated IOPs.

Vision loss after ON degeneration is also a hallmark of glaucoma. We thus tested vision function of 3-month-old mice using ERG recordings. The scotopic b-wave amplitude was reduced at $0.3,3$, and $20 \mathrm{~cd} \mathrm{sec} / \mathrm{m}^{2}$ flash intensities (Fig. S8A-C, G), and the photopic b-wave (Fig. S8D, E, H) and flicker amplitude (Fig. S8F, I) was also reduced in mutant mice, while no difference was observed for the scotopic a-wave amplitude (Fig. S8A-C, G), suggesting impaired visual function in the inner retina. Additionally, the amplitudes of the oscillatory potentials (OPs), which originate from the functional inner retina in mice [37], were also significantly reduced in mutant mice (Fig. S9), reflecting inner retinal dysfunction.

\section{Biochemical analysis of mutant PITX2 proteins}

RT-PCR was performed on mRNA extracted from retina of adult WT mice. Among the three alternatively-spliced murine isoforms (Pitx2a, Pitx2b and Pitx2c) (Fig. S10A), RT-PCR analysis revealed that only Pitx2c isoform was expressed in the mouse retina (Fig. S10B). c. G344T variant was introduced into Pitx2c coding sequence by site-directed mutagenesis to examine the impact of this variant. Western analysis showed unique immune positive bands when probed with anti-PITX2 antibody or Flag-tag antibody (Fig. 5A), suggesting unchanged expression level (Fig. 5B). Immunofluorescence staining revealed that both WT and mutant proteins mainly localized in the nucleus (Fig. 5C). These data demonstrated that this variant is unlikely to impact the expression and cellular localization of the PITX2 protein.

\section{The missense mutation disrupts the interaction of PITX2 with NRF2/YAP1 and impairs the transcriptional activity of PITX2 for antioxidant genes}

Previous studies suggest PITX2 expression and activity depends on NRF2-activated transcription and nuclear shuttling, where PITX2 binds to YAP1 and cooperatively activates transcription of antioxidant genes in mouse myocardium after injury [38]. We reasoned that the interactions of PITX2 with NRF2/YAP1 could also play key roles in the transcription of antioxidant genes under injury condition in the retina. Thus, we analyzed the interactions between WT or mutant PITX2 and NRF2/YAP1 by coimmunoprecipitation, in transiently transfected 293T cells. Upon co-expression of Flag-tagged WT PITX2 and HA-tagged NRF2, a distinct anti-HA signal
(NRF2) was detected in cell lysates as well as Flag precipitates (Fig. $5 \mathrm{D}$, left panel), confirming that PITX2 interacts with NRF2. We similarly confirmed interactions of PITX2 with YAP1 (Fig. 5D, right panel). By contrast, in the presence of mutant PITX2, the anti-HA signal (NRF2) was significantly weakened in Flag precipitates using the same procedure (Fig. 5E, left panel), and that of YAP1 was almost undetectable (Fig. 5E, right panel). Relative quantification (detail in methods) indicated that the binding capacity of mutant PITX2 to NRF2 and YAP1 was reduced $53.78 \%$ and $89.12 \%$ of WT PITX2, respectively (Fig. $6 \mathrm{C}$ ), indicating that the missense mutation disrupts the interaction of PITX2 with NRF2 and YAP1, which might in turn affect its transcriptional activity. We then evaluated the transcriptional activity of the mutant PITX2 by assessing luciferase activity in transfected 293T cells using the LF-2700 promoter constructs [29]. Compared with the empty vector, WT PITX2 activated transcription from the LF-2700 promoter construct by approximately 12-fold (Fig. $5 G)$. By contrast, the mutant PITX2 lost $\sim 69.7 \%$ of its transcriptional activity compared to WT version (Fig. 5G). Thus, the missense mutation impairs the ability of PITX2 protein to recognize and bind to its target sequences and activate transcription of downstream genes. Additionally, to further determine whether the NRF2-YAP1 axis participates in anterior chamber failure in Pit $x 2^{K I}$ animals, ocular cryosections were immunofluorescently labeled using antibodies against NRF2 and YAP1. No significant difference in staining was observed between WT and mutant mice (Fig. S11), implying that other mechanisms may underly the anterior chamber malformations.

We next performed transcriptomic analysis using of retinas from 2-month-old Pitx2 mutant mice and WT littermate controls to obtain insights into the molecular mechanisms. Analyses of the RNA-seq data revealed that expression of the antioxidative response-related genes (Cyp2a5, Upk3b1, Rrm2, Cyp26a1, Plet1, Slc25a24, Cyp2s1, Cyp2f2, Cyp26b1, and Cyp4a12b) were significantly downregulated in Pitx2 mutant retinas (Fig. 5H). These results were further confirmed by real-time quantitative $P C R$ (qPCR) (Fig. 5I). Taken together, based on the transcriptome results, we propose that the rapid RGC loss and ON dystrophy in mutant retina results not only from the elevated IOP caused by anterior segment dysgenesis, but also to the compromised antioxidant capacity caused by impaired PITX2 transcriptional activity of antioxidant genes.

\section{Pitx ${ }^{K l}$ mutant retinas exhibit increased oxidative stress}

Given the impaired PITX2-mediated transcription of antioxidant genes in mutant mice, we next examined the oxidative status of Pitx ${ }^{K I}$ retinas of 6-week-old mice. Several indicators of oxidative stress were evaluated by ELISA using isolated retinal lysates. Compared to controls, decreased activities of the antioxidant 

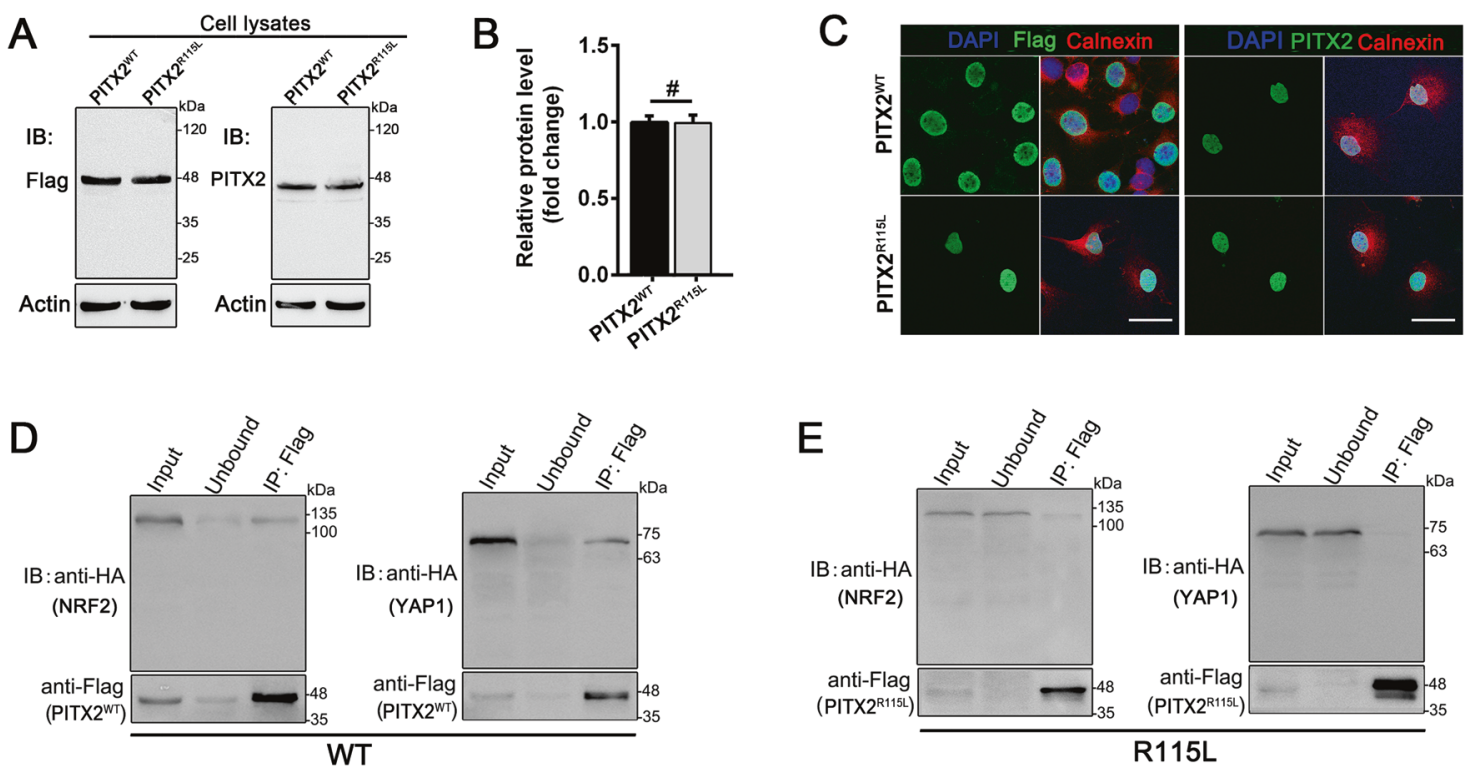

\section{F}
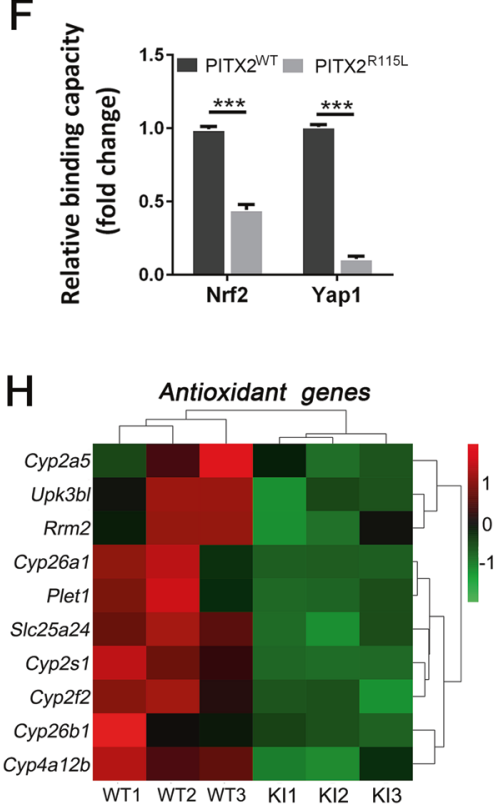

G
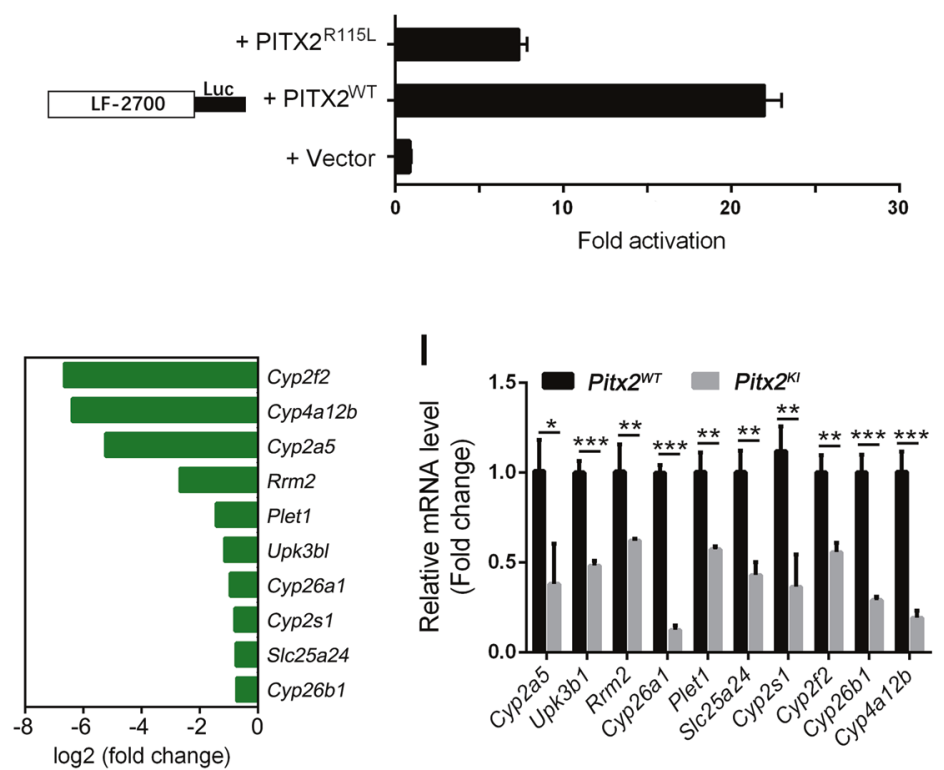

Fig. 5 Analysis of the biochemical and molecular changes observed in Pitx $2^{K I}$ mutant mice. A Expression of WT and mutant PITX2 proteins in 293 T cells. The $p C M V$-Pitx $2^{W T}$ and $p C M V$-Pitx $2^{R 115 L}$ expression plasmids were transfected into $293 T$ cells. Western blot analysis showed mutant PITX2 protein had the same abundance as WT. Antibody of Flag-tag was used to verify the specificity of PITX2 antibody. $\beta$-actin was used as the loading control. B Quantitative comparison of the WT and mutant PITX2 protein levels in transfected cells ( $n=6$ per cohort). C Subcellular localization of WT and mutant PITX2 proteins in COS7 cells. Immunocytochemistry study using anti-PITX2 antibody indicated that both WT and mutant PITX2 proteins were localized in the nucleus. D 293T cells were co-transfected with the indicated constructs. Flag immunoprecipitation, and blotting with anti-HA confirmed physical interaction. PITX ${ }^{W T}$-Flag was immunoprecipitated and the amount of coprecipitated NRF2-HA (left panels) or YAP1-HA (right panels) was determined by immunoblotting, shown are representative immunoblots. E PITX2 ${ }^{\text {R115L }}$-Flag was immunoprecipitated and the amount of co-precipitated NRF2-HA (left panels) or YAP1-HA (right panels) was determined by immunoblotting, shown are representative immunoblots. F Quantification of immunoblots reveals the PITX2 p. R115L mutation decreases its interaction with NRF2 and YAP1 compared to WT ( $n=4$ in each group). G The transcriptional activity of WT and mutant PITX2 was detected with a luciferase reporter in transient transfections of 293T cells. All luciferase activities are normalized to $\beta$-galactosidase activity and shown as mean-fold activation compared with the vector. Five independent replicas were performed. The mean LEF-1 promoter luciferase activity with PITX2 expression was about 200,000 light units per $15 \mu \mathrm{g}$ protein, and the $\beta$-galactosidase activity was about 65,000 light units per $15 \mu \mathrm{g}$ protein. H RNA-seq results showing that several antioxidant-related genes are downregulated in mutant retinas. I RT-qPCR verified the RNA-seq results for the indicated genes $(n=12)$. ${ }^{*} P<0.05 ;{ }^{* *} P<0.01 ;{ }^{* *} P<0.001$. \#, no significant difference. The data are represented as mean \pm SEM.

enzymes superoxide dismutase (SOD) and glutathione peroxidase (GSH-Px) and increased malondialdehyde (MDA) content were observed in mutant retinas (Fig. 6A-C), suggesting compromised antioxidant capacity and peroxidation in mutant retinas.
Retinal cryosections stained with dihydroethidium (DHE) (Fig. 6D) showed increased DHE reaction in $\mathrm{n}$ mutant retinas. Quantification of fluorescence intensity revealed a significant increase in superoxide production in mutant mice, compared to 
A

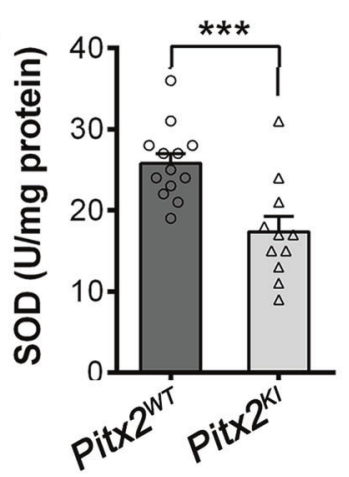

B

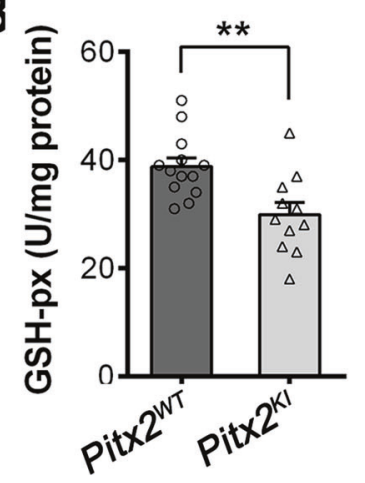

C

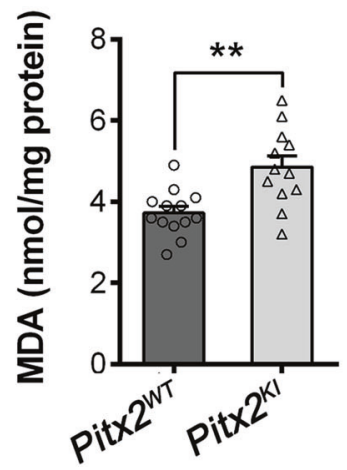

D
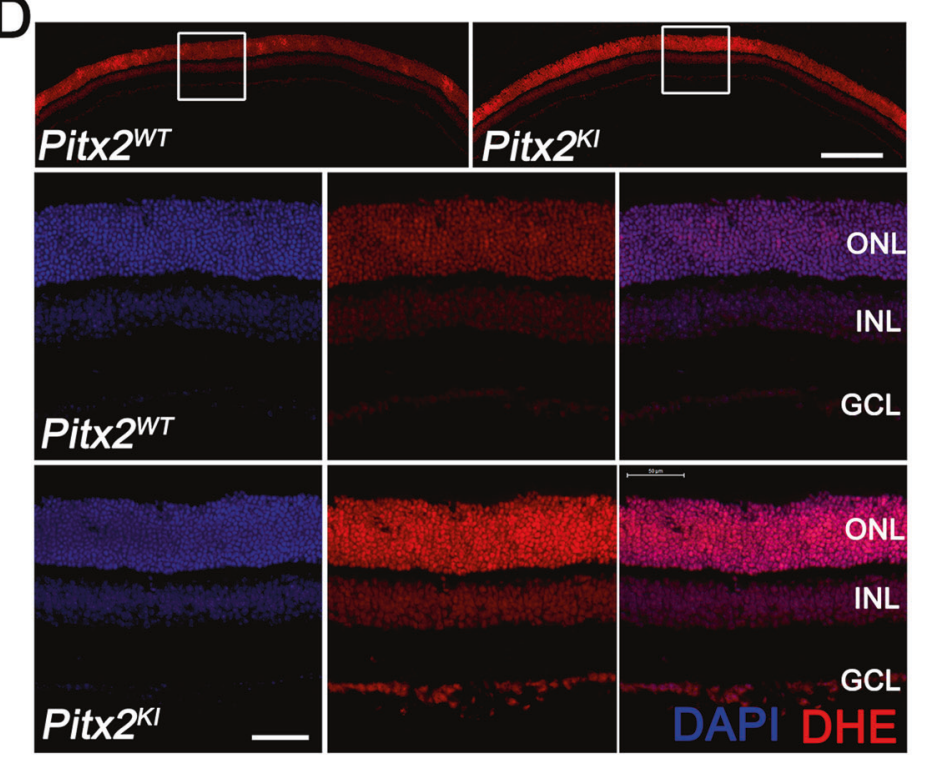

$E$

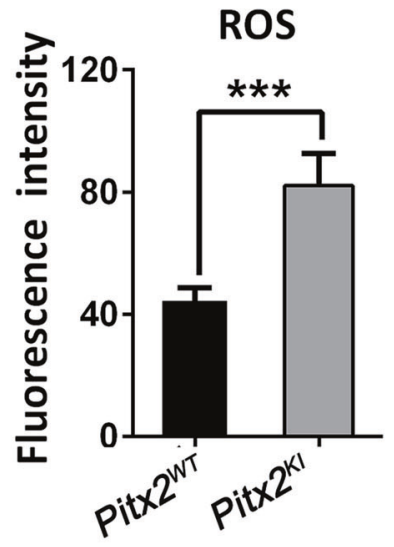

F
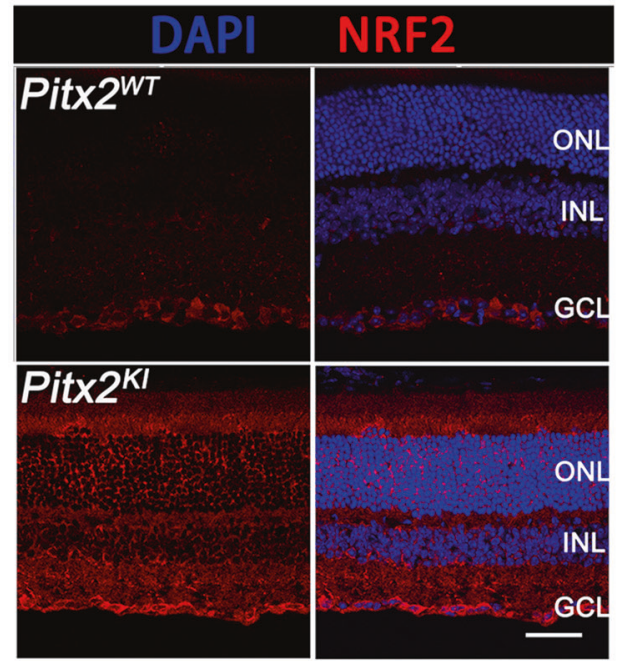

that of controls (Fig. 6E). In addition, both immunofluorescence and western blot demonstrated a significant increase in the NRF2 level in mutant retinas compared to controls (Fig. 6F-H). NRF2 regulates the expression of antioxidant proteins that attenuate cellular oxidative stress, thus its increased expression signifies activation of oxidative stress.
G

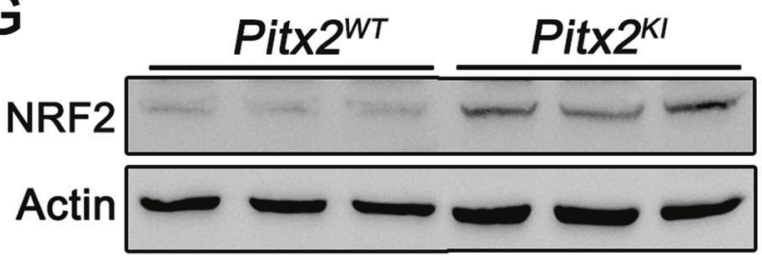

$\mathrm{H}$

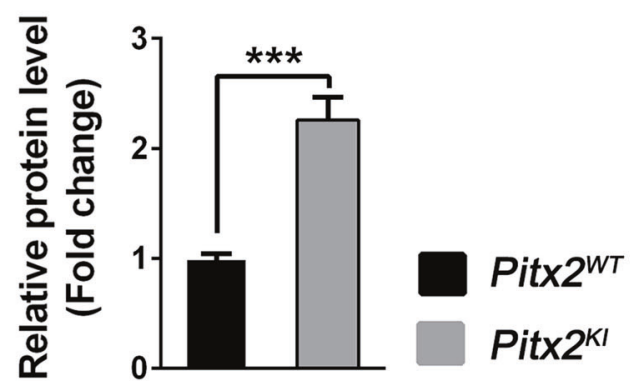

Glial activation and RGC apoptosis in Pitx $2^{K I}$ retinas

We assessed the status of glial activation in mutant retinas from 2month-old mice. In control retinas, CD68 immunoreactivity was weak and punctate, while in mutant retinas staining was intense and agminated in the inner retina (Fig. S12A), suggestive of an activated state. Moreover, the expression level of another 
Fig. 6 Increased oxidative stress in the retinas of Pitx $\mathbf{2}^{\boldsymbol{K} I}$ mice. A-C Mean levels of SOD (A) and GSH-Px (B) activities, MDA (C) content in the Pitx $2^{W T}(n=13)$ and Pit $2^{K I}(n=11)$ retinas. Individual values are shown by dots in the histogram. D ROS activity measured by DHE staining; the red staining is positive for ROS content. Nuclei were counter-stained with DAPI (blue). Scale bars: $200 \mu \mathrm{m}$. High-magnification images of boxed areas are shown in the lower panel. Scale bars: $25 \mu \mathrm{m}$. E Quantification of the fluorescence intensity of DHE staining $(n=9)$. F Representative confocal microscopy images of immunofluorescent staining of NRF2 (oxidative stress regulator, red) and DAPI (blue) in retina sections from 5week-old mice. Scale bar: $25 \mu \mathrm{m}$. G Representative immunoblots of 5-week-old retinal lysates from Pit $\times 2^{W T}$ and Pit $\times 2^{K I}$ mice stained for NRF2. $\beta$-actin was used as the loading control. Uncropped immunoblotting images are shown in Fig. S12. H Quantification of immunoblots of retina lysates from Pitx $2^{W T}$ and Pitx $2^{K I}$ mutant mice. NRF2 level (normalized to control) in retinas from mutant mice was elevated compared with levels in control mice $(n=9)$. ONL, outer nuclear layer; INL, inner nuclear layer; GCL, ganglion cell layer. ${ }^{*} P<0.05 ;{ }^{* *} P<0.01 ;{ }^{* * *} P<0.001$. The data are presented as mean \pm SEM.

microglia marker lba1 was found to be increased in mutant retinas by western blot analysis (Fig. S12B, C). Additionally, activated Müller glia were also distinctly recognizable in mutant retinas by immunostaining with an antibody against glial fibrillary acidic protein (GFAP) (Fig. S12A). These data confirmed astrogliosis in mutant retinas characterized by upregulation of GFAP level relative to controls (Fig. S12B, C). TUNEL assay revealed increased cell apoptosis in the $\mathrm{GCL}$ of mutant retinas, likely due to prolonged oxidative stress and excessive inflammatory reaction (Fig. S12D, E).

\section{Treatment with N-Acetyl-L-cysteine mitigates high intraocular pressure-induced retinal injury by inhibiting oxidative stress in Pitx $2^{K I}$ retinas}

The apparent oxidative stress and subsequent ocular injury prompted us to investigate whether an oxidative reductant would have protective efficacy in Pitx2 mutant mice. N-Acetyl-L-cysteine, an antioxidant, provides increased glutathione and exerts antioxidative protection. Thus, to rescue the RGC degeneration, $\mathrm{N}$-Acetyl-L-cysteine $(25 \mathrm{mg} / \mathrm{kg} /$ day) or PBS (serve as vehicle control), were intraperitoneally administered daily to Pitx2 WT or mutant mice after 5 -weeks of age (Fig. 7A). After 5 weeks of treatment, mice were sacrificed to analysis.

$\mathrm{N}$-Acetyl-L-cysteine-treated mutant mice (hereafter treated) showed no significant differences in IOP from vehicle-treated mutant mice (hereafter vehicle) (Fig. 7B). However, ROS activity was significantly affected in retinas of treated mutant mice. Imaging of vehicle mutant retinas demonstrated an increased DHE reaction in the GCL, INL, and ONL (Fig. $7 \mathrm{C}$ ), while the DHEsuperoxide reaction was largely prevented in the treated mutant retinas, which showed comparable staining intensity to that in WT retina (Fig. 7D). N-Acetyl-L-cysteine administration likely improved the oxidative stress response induced by elevated IOP in mutant retinas.

The RGC loss and ON damage was also evaluated by histological analysis. In vehicle mutant mice, the number of cells in GCL was drastically decreased compared to vehicle-treated WT controls. By contrast, the number of cells retained in the GCL were significantly greater in mutant mice administered N-Acetyl-L-cysteine, compared with corresponding vehicle-treated mice (Fig. 7E, F). The RGC density was further evaluated by immunofluorescent staining of Brn3a of retinal flatmounts. Consistent with H\&E staining, NAcetyl-L-cysteine treatment appeared to increas the number of Brn3a-positive cells in mutant retina compared to that of vehicle mutant mice in all four retinal quadrants (Fig. 7G), but still did not reach WT levels (Fig. $7 \mathrm{H}$ ). To directly monitor the therapeutic effect on RGC death, the RGC number between vehicle- and treatedgroup of each genotype (indicated as $\Delta$ RGC number) were quantified. An increased number of Brn3a-positive cells after $\mathrm{N}$ Acetyl-L-cysteine treatment was observed (Fig. 7I). Together, these data suggested that $\mathrm{N}$-Acetyl-L-cysteine displays significant therapeutic effect on glaucomatous neuropathies through mitigation of oxidative stress and subsequent partial preservation of RGC in Pitx $2^{K I}$ retinas (Fig. 8).

\section{DISCUSSION}

The anatomical and physiological similarity between human and mouse eyes and the ability to genetically manipulate mice make them an excellent model to investigate disease mechanisms and potential intervention of diseases, such as glaucoma. The heritable $\mathrm{DBA} / 2 \mathrm{~J}$ glaucoma model has provided invaluable information on disease cell biology and intervention [39-41]. However, elevated IOP and glaucoma phenotypes do not manifest until 9-12 months, making mechanistic studies and testing of therapeutic strategies expensive and time-consuming. As a novel ENU induced Pitx $2^{\text {eg/1 }}$ and the CRISPR/Cas9 mediated Pitx $2^{K I}$ glaucoma models exhibit early-onset glaucoma, including elevated IOP and RGC degenerative phenotypes., they provide valuable insight into degeneration of RGC and its related molecular pathways. At the molecular level, the PITX2 p.R115L missense mutation disrupts binding of PITX2 to YAP1 and NRF2 and impairs its transcriptional activation of downstream genes involved in regulating oxidative stress (Figs. 5 and 6). Reduced activities of the antioxidant enzymes SOD and GSH-Px were observed in Pitx2 mutant retinas, indicating compromised antioxidant capacity and peroxidation (Fig. 5). In support of this, treatment with N-Acetyl-I-cysteine mitigates the RGC injury observed, presumably by inhibiting oxidative stress in mutant retinas (Fig. 7).

Alterations in the level of functional PITX2 protein (either increased or decreased) may contribute to the pathologies observed [42]. Previous studies reported that heterozygote Pitx2 null mice exhibited thinning of the ventral body, small body size, and ocular and tooth defects [21], whereas in other studies heterozygote alleles do not show obvious haploinsufficient phenotypes [43]. In the present study, heterozygous Pitx ${ }^{K I}$ mutants do not show any obvious glaucomatous phenotype (Fig. S3). This is probably due to the fact that the missense mutation studied here is likely to be a hypomorphic variant (Fig. 5). Additionally, incomplete penetrance was evident in our mouse models, with only $47 \%$ of mutants presenting with elevated IOP at 4 months of age (Fig. 3C), which is consistent with the observation that only $50 \%$ of patients with ARS develop elevated IOP and glaucoma. Moreover, while teeth hypodontia or microdontia is reported in some human ARS patients and not others, no apparent non-ocular abnormalities were observed in adult Pit $\times 2^{K l}$ mice. Thus, our mutants model recapitulates ocular phenotypes of ARS patients with PITX2 mutations [16, 44, 45].

We demonstrate two possible pathological mechanisms that underlie the glaucoma phenotypes induced by the missense mutation in Pitx2 (Fig. 8). The observed anterior segment dysplasia in Pitx $2^{K l}$ mice likely contributes to the dysfunction of the drainage system leading to an elevation in IOP, which serves as an initiator of the disease process. The mutation also exacerbates the high IOPinduced oxidative stress in the retina by disrupting the interaction of PITX2 with NRF2/YAP1, which consequently impairs the transcription of several antioxidant genes. The reduction of antioxidant capacity may accelerate RGC loss and optic neuropathy. The anterior segment dysplasia caused by Pitx2 variation has been well documented, but the precise mechanism through which subsequent pathogenesis occurs remains unclear. The present study 
A

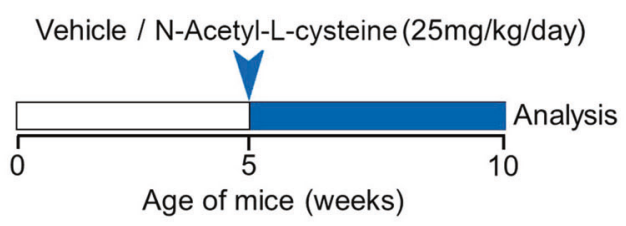

Intraperitoneal injection
B

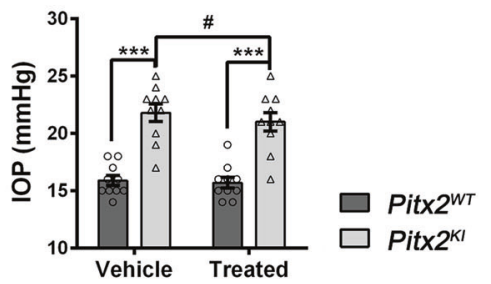

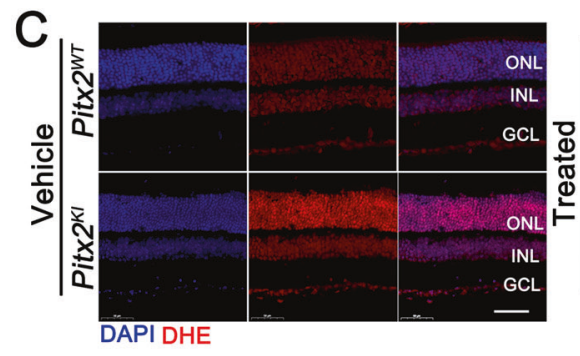

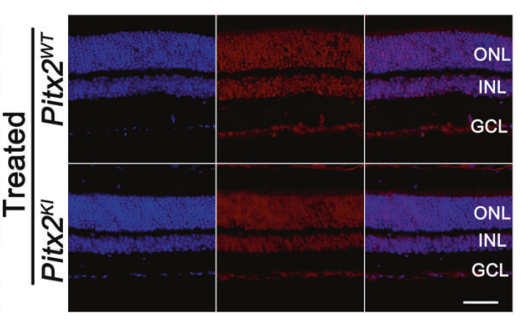

$E$

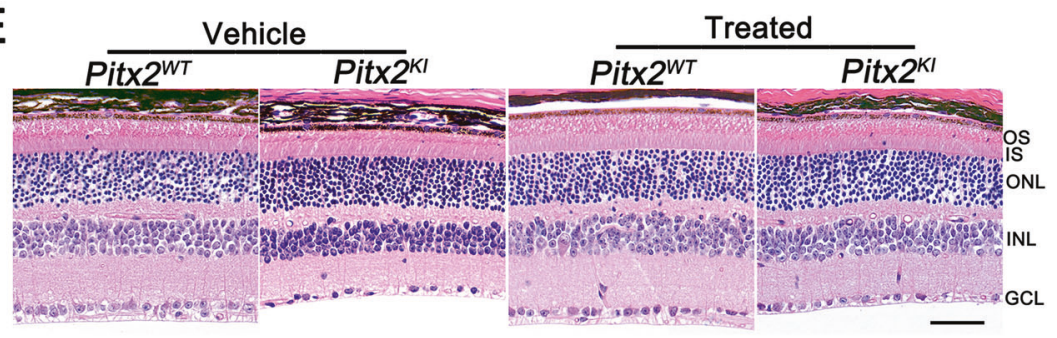

D

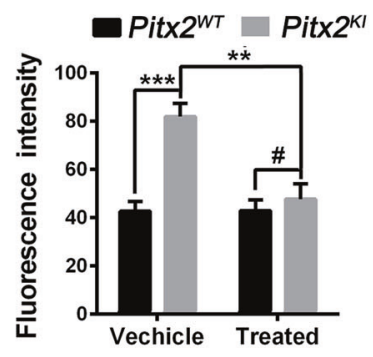

F

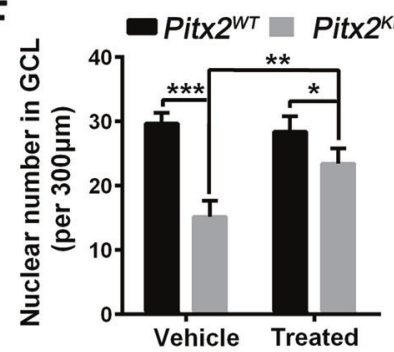

G

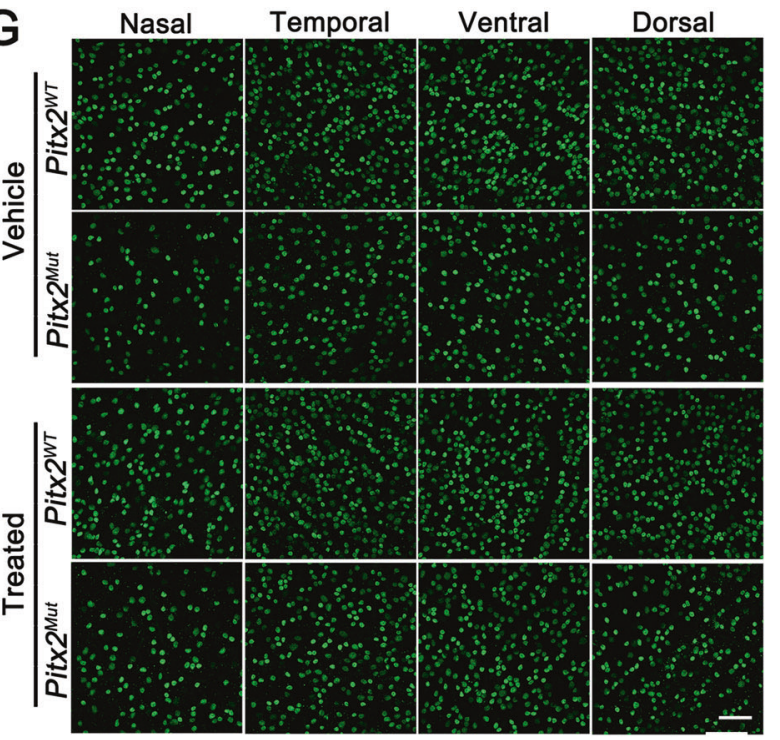

mainly focuses on the latter, revealing a novel function of PITX2 in RGCs and in glaucomatous pathogenesis. We demonstrated that PITX2 moderates oxidative stress response through the NRF2-YAP1 axis in RGCs, whereas according to immunostaining data, this mechanism does not appear to be involved in the early post-natal phenotype (Fig. S11). The potential mechanisms triggering the anterior segment dysgenesis warrant further investigation.

Owing to the high demand of oxygen and energy necessary to generate action potentials, RGCs contain large number of

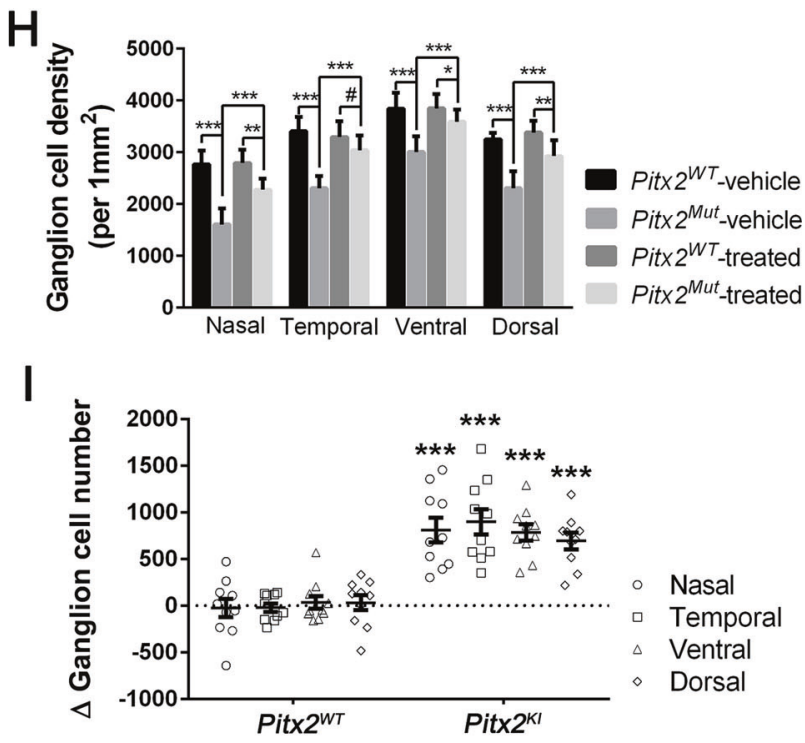

mitochondria throughout the cell soma, axon and dendrites $[46,47]$. Moreover, mitochondrial dysfunction has been recognized to be relevant to the development of glaucoma [48-52]. Transcriptional analysis of DBA/2J glaucoma model revealed mitochondrial dysfunction and metabolite depletion as a primary cause of neuronal damage in glaucoma [53]. These studies suggested that mitochondria dysfunction and oxidative stress play important roles in glaucoma. In our Pit $2^{K I}$ early-onset glaucoma model, elevated expression levels of NRF2, a regulator of cellular 
Fig. 7 Treatment with N-Acetyl-L-cysteine prevents elevated intraocular pressure-induced retinal injury by inhibiting oxidative stress in Pitx $2^{K I}$ mutant retinas. A N-Acetyl-I-cysteine in $40 \%$ PBS was injected intraperitoneally $\left(25 \mathrm{mg} / \mathrm{kg} /\right.$ day) into Pitx $2^{W T}$ and Pitx $2^{K I}$ mutant mice for 5 weeks (from 5-week to 10-weeks of age) as the treated groups. Equivalent volumes of $40 \%$ PBS were delivered intraperitoneally to the Pitx $2^{W T}$ and Pitx $2^{K I}$ mice as the vehicle-treated control groups. All animals were sacrificed at 10 weeks of age to conduct analyses. B IOP analysis in Pit $\times 2^{W T}$ and Pit $\times 2^{K l}$ mice subjected to vehicle and N-Acetyl-I-cysteine treatment. Individual IOP values are shown by dots in the histograms ( $n=10$ in each group). C ROS activity was measured by DHE staining of retinal sections from Pitx $2^{W T}$ and Pitx $2^{K l}$ mutant mice subjected to vehicle or N-acetyl-I-cysteine treatment. The red staining is positive for ROS content. Nuclei were counter-stained with DAPI (blue). Scale bars: $50 \mu \mathrm{m}$. D Quantification of the fluorescence intensity of DHE staining showed an obvious decrease in ROS formation in retinas from treatedPitx $2^{K I}$ mutant mice $\left(n=10\right.$ in each group). E Representative images of H\&E staining of retinal section from Pitx $2^{W T}$ and Pitx $2^{K I}$ mice subjected to vehicle or N-Acetyl-I-cysteine treatment. Scale bars: $25 \mu \mathrm{m}$. OS, outer segment; IS, inner segment; ONL, outer nuclear layer; INL, inner nuclear layer; GCL, ganglion cell layer. $\mathbf{F}$ Quantification of the nuclei number in $\mathrm{GCL}$ shows that the Pitx2 ${ }^{K I}$ mutants have significantly decreased number of nuclei in the GCL ( $n=6$ in each group). G Immunofluorescent enface view of retinal flatmounts from vehicle- or N-Acetyl-Icysteine-treated Pitx $2^{W T}$ and Pit $x 2^{K I}$ mice following labeling with anti-Brn3a to detect RGC. Scale bars: $25 \mu \mathrm{m}$. $\mathbf{H}$ Quantification of the number of RGC showed overall decreased number of RGL Brn3a positve cells. However, comparison of Pitx $2^{K I}$ vehicle group, Pit $\times 2^{K I}$ treated group showed significantly more RGC remaining in N-Acetyl-I-cysteine-treated Pit $2^{K I}$ mice ( $n=12$ in each group). I Quantitation indicates that an obvious increase in $\Delta$ RGC number in Pitx ${ }^{K l}$ retinas ( $n=10$ in each group). $\Delta$ RGC number $=$ RGC number (Treated group)-RGC number (vehicle group). A positive number indicates an increase in RGC number after N-Acetyl-I-cysteine treatment. ${ }^{*} P<0.05 ;{ }^{* *} P<0.01 ;{ }^{* * *} P<0.001$. \#, not significant. The data are presented as mean \pm SEM.

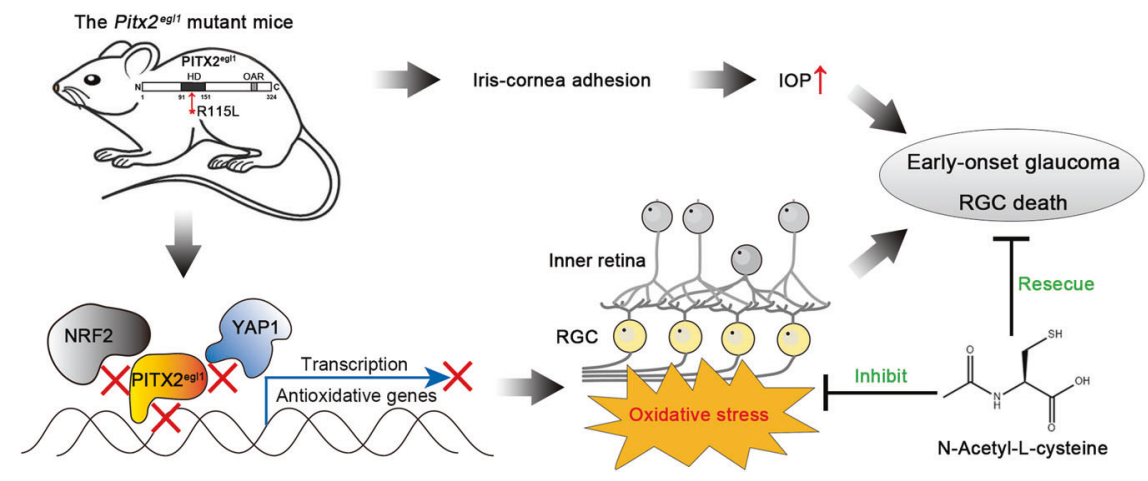

Fig. 8 Schematic diagram of disease mechanism and therapy strategy for Pitx ${ }^{\mathbf{e g l}}$ mutant mice. A novel missense mutation p.R115L in Pitx2 (named Pitx $2^{\text {egli }}$ ) led to iris-cornea adhesion and subsequent elevated intraocular pressure (IOP) accompanied with progressive death of retinal ganglion cells (RGC). These ocular phenotypes recapitulated hallmark features of the human glaucomatous pathologies. Mutant PITX2 protein loses its ability to bind NRF2 (regulated PITX2 expression and nuclei localization) and YAP1 (co-initiate transcription of their downstream targets) and consequently, PITX2-mediated transcription of several antioxidant genes is impaired, hence increasing oxidative stress in the inner retina. Treatment with $\mathrm{N}$-Acetyl-I-cysteine exerted a profound neuroprotective effect on glaucomatous neuropathies associated with the model through inhibition of oxidative stress.

resistance to oxidative stress, was observed (Fig. 6F-H). N-acetyl-Lcysteine treatment mitigated RGC loss by inhibiting oxidative stress in Pitx2 mutant retinas (Figs. 7 and 8). Cumulatively, our data identifies a potential target for development of therapeutic strategies for ARS induced glaucoma and also, a potential strategy for glaucoma therapy development.

\section{DATA AVAILABILITY}

All data generated or analyzed during this study are included in this published article and its supplementary information files. The data sets analyzed during the current study are available in Repository with Accession ID: CRA005112, Databank URL http:// ngdc.cncb.ac.cn/gsa.

\section{REFERENCES}

1. Quigley HA, Addicks EM, Green WR, Maumenee A. Optic nerve damage in human glaucoma: II. The site of injury and susceptibility to damage. Arch Ophthalmol. 1981;99:635-49.

2. Nickells RW, Howell GR, Soto I, John SW. Under pressure: cellular and molecular responses during glaucoma, a common neurodegeneration with axonopathy. Annu Rev Neurosci. 2012;35:153-79.

3. Coleman AL, Miglior S. Risk factors for glaucoma onset and progression. Surv Ophthalmol. 2008;53:S3-S10.

4. Becker B. Tonography in the diagnosis of simple (open angle) glaucoma. Trans Am Acad Ophthalmol Otolaryngol. 1961;65:156-62.

5. Fautsch MP, Johnson DH, Group SAPRIW. Aqueous humor outflow: what do we know? Where will it lead us? Investig Ophthalmol Vis Sci. 2006;47:4181-7.
6. Liu Y, Allingham RR. Molecular genetics in glaucoma. Exp Eye Res. 2011;93:331-9.

7. Cascella R, Strafella C, Germani C, Novelli G, Ricci F, Zampatti S, et al. The genetics and the genomics of primary congenital glaucoma. BioMed Res Int. 2015;2015:321291.

8. Cvekl A, Tamm ER. Anterior eye development and ocular mesenchyme: new insights from mouse models and human diseases. Bioessays. 2004;26:374-86.

9. Gage PJ, Zacharias AL. Signaling "cross-talk" is integrated by transcription factors in the development of the anterior segment in the eye. Developmental Dyn: Off Publ Am Assoc Anatomists. 2009;238:2149-62.

10. Gould DB, Smith RS, John SW. Anterior segment development relevant to glaucoma. Int J Developmental Biol. 2004;48:1015-29.

11. Ito YA, Walter MA. Genomics and anterior segment dysgenesis: a review. Clin Exp Ophthalmol. 2014;42:13-24.

12. Sowden J. Molecular and developmental mechanisms of anterior segment dysgenesis. Eye. 2007;21:1310-8.

13. Reis LM, Tyler RC, Kloss BAV, Schilter KF, Levin AV, Lowry RB, et al. PITX2 and FOXC1 spectrum of mutations in ocular syndromes. Eur J Hum Genet. 2012;20:1224-33.

14. Tümer Z, Bach-Holm D. Axenfeld-Rieger syndrome and spectrum of PITX2 and FOXC1 mutations. Eur J Hum Genet. 2009;17:1527-39.

15. Footz T, Idrees F, Acharya M, Kozlowski K, Walter MA. Analysis of mutations of the PITX2 transcription factor found in patients with Axenfeld-Rieger syndrome. Investig Ophthalmol Vis Sci. 2009;50:2599-606.

16. Semina EV, Reiter R, Leysens NJ, Alward WLM, Small KW, Datson NA, et al. Cloning and characterization of a novel bicoid-related homeobox transcription factor gene, RIEG, involved in Rieger syndrome. Nat Genet. 1996;14:392-9.

17. Kulak SC, Kozlowski K, Semina EV, Pearce WG, Walter MA. Mutation in the RIEG1 gene in patients with iridogoniodysgenesis syndrome. Hum Mol Genet. 1998;7:1113-7. 
18. Alward WL, Semina EV, Kalenak JW, Héon E, Sheth BP, Stone EM, et al. Autosomal dominant iris hypoplasia is caused by a mutation in the Rieger syndrome (RIEG/ PITX2) gene. Am J Ophthalmol. 1998;125:98-100.

19. Shields MB. Axenfeld-Rieger syndrome: a theory of mechanism and distinctions from the iridocorneal endothelial syndrome. Trans Am Ophthalmological Soc. 1983;81:736.

20. Noden D. Periocular mesenchyme: neural crest and mesodermal interactions. Duane's Fundations Clin Ophthalmol. 1993;1:1-23.

21. Chen L, Gage PJ. Heterozygous Pitx2 null mice accurately recapitulate the ocular features of Axenfeld-Rieger syndrome and congenital glaucoma. Investigative Ophthalmol Vis Sci. 2016;57:5023-30.

22. Yang Y, Liu W, Sun K, Jiang L, Zhu X. Tmem30a deficiency leads to retinal rod bipolar cell degeneration. J Neurochem. 2019;148:400-12.

23. Truett G, Heeger P, Mynatt R, Truett A, Walker J, Warman M. Preparation of PCRquality mouse genomic DNA with hot sodium hydroxide and tris (HotSHOT). Biotechniques. 2000;29:52-4.

24. Taylor BA, Navin A, Phillips SJ. PCR-amplification of simple sequence repeat variants from pooled DNA samples for rapidly mapping new mutations of the mouse. Genomics. 1994;21:626-32.

25. Fairfield H, Gilbert GJ, Barter M, Corrigan RR, Curtain M, Ding Y, et al. Mutation discovery in mice by whole exome sequencing. Genome Biol. 2011;12:1-12.

26. Li X, Florez S, Wang J, Cao H, Amendt BA. Dact2 represses PITX2 transcriptional activation and cell proliferation through $\mathrm{Wnt} /$ beta-catenin signaling during odontogenesis. PLoS ONE. 2013;8:e54868.

27. Wang J, Sun Z, Zhang Z, Saadi I, Wang J, Li X, et al. Protein inhibitors of activated STAT (Pias1 and Piasy) differentially regulate pituitary homeobox 2 (PITX2) transcriptional activity. J Biol Chem. 2013;288:12580-95.

28. Vadlamudi U, Espinoza HM, Ganga M, Martin DM, Liu X, Engelhardt JF, et al. PITX2, $\beta$-catenin and LEF-1 interact to synergistically regulate the LEF-1 promoter. J Cell Sci. 2005;118:1129-37.

29. Filali $M$, Cheng $N$, Abbott $D$, Leontiev V, Engelhardt JF. Wnt-3A/ $\beta$-catenin signaling induces transcription from the LEF-1 promoter. J Biol Chem. 2002;277:33398-410.

30. Arany ZP. High-throughput quantitative real-time PCR. Curr Protoc Hum Genet. 2008;58:11.10. 11-11.10. 11

31. Du Y, Veenstra A, Palczewski K, Kern TS. Photoreceptor cells are major contributors to diabetes-induced oxidative stress and local inflammation in the retina. Proc Natl Acad Sci USA. 2013;110:16586-91.

32. Kipfer-Kauer A, McKinnon SJ, Frueh BE, Goldblum D. Distribution of amyloid precursor protein and amyloid- $\beta$ in ocular hypertensive C57BL/6 mouse eyes. Curr Eye Res. 2010;35:828-34.

33. Smith RS, Zabaleta A, Kume T, Savinova OV, Kidson SH, Martin JE, et al. Haploinsufficiency of the transcription factors FOXC1 and FOXC2 results in aberrant ocular development. Hum Mol Genet. 2000;9:1021-32.

34. Priston M, Kozlowski K, Gill D, Letwin K, Buys $Y$, Levin AV, et al. Functional analyses of two newly identified PITX2 mutants reveal a novel molecular mechanism for Axenfeld-Rieger syndrome. Hum Mol Genet. 2001;10:1631-8.

35. Kozlowski K, Walter MA. Variation in residual PITX2 activity underlies the phenotypic spectrum of anterior segment developmental disorders. Hum Mol Genet. 2000;9:2131-9.

36. Evans AL, Gage PJ. Expression of the homeobox gene Pitx2 in neural crest is required for optic stalk and ocular anterior segment development. Hum Mol Genet. 2005;14:3347-59.

37. Heynen $\mathrm{H}$, Wachtmeister $\mathrm{L}$, van Norren D. Origin of the oscillatory potentials in the primate retina. Vis Res. 1985;25:1365-73.

38. Tao G, Kahr PC, Morikawa Y, Zhang M, Rahmani M, Heallen TR, et al. Pitx2 promotes heart repair by activating the antioxidant response after cardiac injury. Nature. 2016;534:119-23.

39. Chang B, Smith RS, Hawes NL, Anderson MG, Zabaleta A, Savinova O, et al Interacting loci cause severe iris atrophy and glaucoma in DBA/2J mice. Nat Genet. 1999;21:405-9.

40. John S, Smith RS, Savinova OV, Hawes NL, Chang B, Turnbull D, et al. Essential iris atrophy, pigment dispersion, and glaucoma in DBA/2J mice. Investig Ophthalmol Vis Sci. 1998;39:951-62.

41. Libby RT, Anderson MG, Pang I-h, Robinson ZH, Savinova OV, Cosma IM, et al. Inherited glaucoma in DBA/2J mice: pertinent disease features for studying the neurodegeneration. Vis Neurosci. 2005;22:637.

42. Gage PJ, Suh H, Camper SA. Dosage requirement of Pitx2 for development of multiple organs. Development. 1999;126:4643-51.

43. Lu M-F, Pressman C, Dyer R, Johnson RL, Martin JF. Function of Rieger syndrome gene in left-right asymmetry and craniofacial development. Nature. 1999;401:276-8.

44. Amendt B, Semina E, Alward W. Rieger syndrome: a clinical, molecular, and biochemical analysis. Cell Mol Life Sci CMLS. 2000;57:1652-66.

45. Strungaru MH, Dinu I, Walter MA. Genotype-phenotype correlations in AxenfeldRieger malformation and glaucoma patients with FOXC1 and PITX2 mutations. Investig Ophthalmol Vis Sci. 2007;48:228-37.

46. Harris JJ, Attwell D. The energetics of CNS white matter. J Neurosci. 2012;32:356-71.
47. Li Z, Okamoto K-I, Hayashi Y, Sheng M. The importance of dendritic mitochondria in the morphogenesis and plasticity of spines and synapses. Cell. 2004;119:873-87.

48. Chrysostomou V, Rezania F, Trounce IA, Crowston JG. Oxidative stress and mitochondrial dysfunction in glaucoma. Curr Opin Pharmacol. 2013;13:12-15.

49. Kong GY, Van Bergen NJ, Trounce IA, Crowston JG. Mitochondrial dysfunction and glaucoma. J Glaucoma. 2009;18:93-100.

50. Lee S, Van Bergen NJ, Kong GY, Chrysostomou V, Waugh HS, O'Neill EC, et al. Mitochondrial dysfunction in glaucoma and emerging bioenergetic therapies. Exp Eye Res. 2011;93:204-12.

51. Lascaratos G, Garway-Heath DF, Willoughby CE, Chau K-Y, Schapira AH. Mitochondrial dysfunction in glaucoma: understanding genetic influences. Mitochondrion. 2012;12:202-12.

52. Inman DM, Harun-Or-Rashid M. Metabolic vulnerability in the neurodegenerative disease glaucoma. Front Neurosci. 2017;11:146

53. Williams PA, Harder JM, Foxworth NE, Cochran KE, Philip VM, Porciatti V. Vitamin B3 modulates mitochondrial vulnerability and prevents glaucoma in aged mice. Science. 2017:355:756-60.

\section{ACKNOWLEDGEMENTS}

The authors want to thank Chengdu LiLai Biotechnology Co. Ltd for technical assistance with histology analysis and transmission electron microscopy service. This study was supported by: The National Natural Science Foundation of China (82121003, 81970841, and 81770950 to Xianjun Zhu, 82101160 to Yeming Yang, and 81770924, 82070963, to Xuyang Liu), and the Department of Science and Technology of Sichuan Province (21ZDYF4279, 2020ZYD037, 2020ZYD038, 2020JDZH0026, 2021JDZH0022), the CAMS Innovation Fund for Medical Sciences (2019-12M-5-032), and US National Institutes of Health (NIH) Grant EY019943 and EY027305. The Jackson Laboratory institutional shared services are supported in part by NIH National Cancer Institute Support Grant CA34196. The funders had no role in the study design, data collection and analysis, or preparation of the manuscript.

\section{AUTHOR CONTRIBUTIONS}

$X Z, B C$, and $X$ Liu conceived the project. $X Z$ and $Y Y$ designed experiments. $Y Y, X L i$ JW, JT, BF, PMN, KS, WT, WL, and BC acquired data. $Y Y, B C$, and $X Z$ analyzed data. $Y Y$ and $X Z$ interpreted data. $Y Y$ and $X Z$ drafted and revised the article. $B C, X$ Liu, and $\mathrm{PMN}$ revised manuscript. All authors approved submission for publication.

\section{COMPETING INTERESTS}

The authors declare no competing interests.

\section{ADDITIONAL INFORMATION}

Supplementary information The online version contains supplementary material available at https://doi.org/10.1038/s41419-021-04331-1.

Correspondence and requests for materials should be addressed to Xuyang Liu, Bo Chang or Xianjun Zhu.

Reprints and permission information is available at http://www.nature.com/ reprints

Publisher's note Springer Nature remains neutral with regard to jurisdictional claims in published maps and institutional affiliations.

Open Access This article is licensed under a Creative Commons Attribution 4.0 International License, which permits use, sharing, adaptation, distribution and reproduction in any medium or format, as long as you give appropriate credit to the original author(s) and the source, provide a link to the Creative Commons license, and indicate if changes were made. The images or other third party material in this article are included in the article's Creative Commons license, unless indicated otherwise in a credit line to the material. If material is not included in the article's Creative Commons license and your intended use is not permitted by statutory regulation or exceeds the permitted use, you will need to obtain permission directly from the copyright holder. To view a copy of this license, visit http://creativecommons. org/licenses/by/4.0/. 